\title{
Kodifikationsidee und Europäisches Privatrecht
}

\author{
Eva-Maria Kieninger
}

A. Aufstieg und Fall der Idee eines Europäischen Zivilgesetzbuchs....

I. Die Praxis der Harmonisierungsrichtlinien

II. Die Idee eines Europäischen Zivilgesetzbuchs - von den Resolutionen des Europäischen Parlaments zum DCFR

III. Vom DCFR über die Horizontalrichtlinie zum optionalen europäischen Kaufrecht oder: „Parturient montes, nascetur ridiculus mus"

1. Verbraucherrechterichtlinie ...... 415

2. Der Kommissionsvorschlag für ein gemeinsames Europäisches Kaufrecht....

B. Vom Römer Übereinkommen 1980 zur Idee einer Europäischen IPR-Kodifikation.

I. Die Ablösung der Staatsverträge durch die „Rom“- und „Brüssel“Verordnungen
II. Eine Europäische IPR-Kodifikation

am Horizont? ..................... 423

C. Vergleich............................ 427

I. Kontinuität ........................ 427

II. Rechtsprechung des EuGH ......... 428

III. Immanente Beschränkung auf grenzüberschreitenden Sachverhalte ..... 429

IV. IPR als politikfernes Spezialistenfach ............................ 429

V. Koordinierung des Privatrechts durch vereinheitlichtes IPR und IZVR als Ausdruck einer noch unvollkommenen Europäischen Union 430

In einer kürzlich publizierten, weit ausholenden Studie über die Zukunft der Kodifikationsidee schreibt Frédéric Zenati-Castaing:

"L'aventure moderne de la codification ne s'arrête pas aux codes nationaux. Le déclin de nations comme cadres de systèmes juridiques ne veut pas dire que la codification de type moderne ait achevé de remplir son rôle et se trouve frappée de péremption. Une nouvelle mission lui est assignée par la transformation que subissent les ordres juridiques dans les temps modernes et postmodernes, celle d'instrument irremplaçable d'unification."1

Die Kodifikationsidee ${ }^{2}$ ist danach keineswegs überholt, sondern hat als unverzichtbares Mittel der internationalen, weltweiten, aber auch regionalen (Privat-) Rechtsvereinheitlichung eine neue Mission zu erfüllen. Misst man diese hochgestimmte Vision an der Realität, so stimmt die Geschichte der bisherigen Bemühungen um eine Kodifikation des Privatrechts auf europäischer Ebene allerdings eher skeptisch (A.).

* Prof. Dr. Eva-Maria Kieninger ist Inhaberin des Lehrstuhls für deutsches und europäisches Privatrecht sowie Internationales Privatrecht der Universität Würzburg.

1 F. Zenati-Castaing, L'avenir de la codification, Revue internationale de droit comparé 2011, S. 355 (369f.).

2 Vgl. J. P. Schmidt, Kodifikation, in: J. Basedow/K. J. Hopt/R.Zimmermann (Hrsg.), Handwörterbuch des Europäischen Privatrechts, München 2009, S. 986. 
Erfolgversprechender erscheint die Mission einer Kodifikation des europäischen Internationalen Privatrechts (B.). Mögliche Ursachen werden unter C. erörtert.

\section{A. Aufstieg und Fall der Idee eines Europäischen Zivilgesetzbuchs}

Die Geschichte der Idee eines Europäischen Zivilgesetzbuches setzte 1989 mit einem Paukenschlag ein: Das Europäische Parlament forderte darin den Rat, die Kommission und die Mitgliedstaaten auf, „mit den erforderlichen Vorbereitungsarbeiten zur Ausarbeitung eines einheitlichen Europäischen Gesetzbuchs für das Privatrecht [zu beginnen]".3 1994 erneuerte das Parlament seinen Appell und bat nunmehr die Kommission, „die Arbeiten im Zusammenhang mit der möglichen Ausarbeitung eines einheitlichen Europäischen Gesetzbuches für das Privatrecht in Angriff zu nehmen". 4

\section{Die Praxis der Harmonisierungsrichtlinien}

Bis zu diesem Zeitpunkt war das Privatrecht nur punktuell Objekt von Angleichungsmaßnahmen gewesen. ${ }^{5}$ Verschiedene Harmonisierungsrichtlinien zu Einzelaspekten des Vertragsrechts, vor allem des Verbrauchervertragsrechts, bewirkten eine Mindestangleichung von Widerrufsrechten bei Haustürgeschäften und im Fernabsatz, ${ }^{6}$ von Rechten der Gläubiger im Fall der verzögerten Zahlung durch Unternehmen, ${ }^{7}$ von Ausgleichsansprüchen der Handelsvertreter ${ }^{8}$ und von anderen sektoriell begrenzten Vorschriften. Die ersten beiden Richtlinien, die tiefer in das Gefüge der nationalen Zivilrechtsordnungen hätten hineinwirken können bzw. hineinwirkten, waren die Richtlinie über missbräuchliche Vertragsklauseln $(1993)^{9}$ und die

3 Entschließung des Europäischen Parlaments über die Angleichung des Privatrechts der Mitgliedstaaten vom 26.5.1989, ABl. C 158/400 v. 26.6.1989, abgedruckt in ZEuP 1993, S. 613 ff.

4 Entschließung des Europäischen Parlaments zur Angleichung bestimmter Rechtsbereiche des Privatrechts der Mitgliedstaaten v. 6.5.1994, ABl. C 205/518 v. 25.7.1994, abgedruckt in ZEuP 1995, S. 669; dazu W. Tilmann, Zweiter Kodifikationsbeschluß des Europäischen Parlaments, ZEuP 1995, S. 534 ff. $\mathrm{Zu}$ den Initiativen des Europäischen Parlaments im Hinblick auf ein Europäisches Zivilgesetzbuch gehört auch die von der Generaldirektion Wissenschaft des Europäischen Parlaments herausgegebene „Untersuchung der Privatrechtsordnungen der EU im Hinblick auf Diskriminierungen und die Schaffung eines Europäischen Zivilgesetzbuches“ (1999). Vgl. außerdem die Entschließung des Europäischen Parlaments v. 16.3.2000, ABl. C 377/323 v. 29.12.2000, in der das Europäische Parlament die Auffassung vertritt, "daß eine verstärkte Harmonisierung im Bereich des bürgerlichen Rechts im Binnenmarkt unerlässlich geworden ist" und die Kommission auffordert, "eine diesbezügliche Studie auszuarbeiten".

5 Die folgenden Ausführungen beschränken sich auf vertragsrechtsangleichende Richtlinien. Sie sparen insbesondere die Rechtsangleichungsmaßnahmen im Gesellschafts-, Arbeits-, und Lauterkeitsrecht aus.

6 RL 85/577/EWG betreffend den Verbraucherschutz im Falle von außerhalb von Geschäftsräumen geschlossenen Verträgen v. 20.12.1985, ABl. EG Nr. L 372/31; RL 97/7/EG v. 20.5.1997 über den Verbraucherschutz bei Vertragsabschlüssen im Fernabsatz, ABl. EG Nr. L 144/19, bedie jetzt zusammengefasst in der RL 2011/83/EU v. 25.10.2011 über die Rechte der Verbraucher, ABl. Nr. L 304/64.

7 RL 2011/7/EU v. 16.2.2011 zur Bekämpfung von Zahlungsverzug im Geschäftsverkehr (Neufassung), ABl. EU Nr. L 48/1.

8 RL 86/653/EWG v. 18.12.1986 zur Koordinierung der Rechtsvorschriften der Mitgliedstaaten betreffend die selbständigen Handelsvertreter, ABl. EG Nr. L 382/17.

9 RL 93/13/EWG v. 5.4.1993 über mißbräuchliche Klauseln in Verbraucherverträgen, ABl. EG Nr. L $95 / 29$. 
Verbrauchsgüterkaufrichtlinie (1999). ${ }^{10}$ Die erste hätte mit ihrer $\$ 307$ BGB nachempfundenen Generalklausel das Potenzial gehabt, die mitgliedstaatlichen Rechtsordnungen in einem Kernbereich des Vertragsrechts zumindest in Bezug auf Verbrauchergeschäfte wesentlich stärker anzugleichen als jede ihrer Vorgängerinnen. Hätte der EuGH seine Befugnisse zur konkretisierenden Auslegung des Begriffs der „Missbräuchlichkeit“ von AGB intensiv genutzt, so wäre daraus eine Standardisierung der Vertragspraxis über die mitgliedstaatlichen Grenzen hinweg entstanden. In der deutschen Literatur wurde diese Perspektive allerdings überwiegend als abschreckend empfunden; die Furcht vor dem EuGH als einer zivilrechtlichen Superrevisionsinstanz machte die Runde. ${ }^{11}$ Der EuGH hat die durch die Richtlinie eröffneten Chancen allerdings nicht genutzt bzw. - in den Augen der Kritiker - löbliches judicial self restraint geübt. Er beschränkt sich seit vielen Jahren auf ganz allgemeine Aussagen zum Gehalt der Generalklausel und überlässt die Beurteilung der Missbräuchlichkeit konkreter Klauseln den nationalen Gerichten. ${ }^{12}$ Eine europaweite Standardisierung allgemeiner Geschäftsbedingungen ist auf diese Weise gerade nicht entstanden. ${ }^{13}$

Die Verbrauchsgüterkaufrichtlinie hat das deutsche Recht wesentlich stärker geprägt: Sie wurde zum Anlass für die umfassendste Reform des BGB seit dessen Inkrafttreten. Nicht nur das Kaufrecht, auch das allgemeine Leistungsstörungs- und das Verjährungsrecht wurden durch das am 1.1.2002 in Kraft getretenen Schuldrechtsmodernisierungsgesetz neu gestaltet. ${ }^{14} \mathrm{Ob}$ die damalige Entscheidung des deutschen Gesetzgebers zur Umsetzung der Richtlinie im allgemeinen Kaufrecht richtig war, wird zunehmend in Frage gestellt. Da der EuGH bei der Auslegung der Richtlinie dem Verbraucherschutzgedanken die oberste Priorität einräumt, etwa bei der

10 RL 1999/44/EG v. 25.5.1999 zu bestimmten Aspekten des Verbrauchsgüterkaufs und der Garantien für Verbrauchsgüter, ABl. EG Nr. L 171/12.

11 Vgl. C.-W. Canaris, Der EuGH als zukünftige privatrechtliche Superrevisionsinstanz, EuZW 1994, 417.

12 Ständige Rechtsprechung seit EuGH 1.4.2004 - Rs. C-237/02 (Freiburger Kommunalbauten GmbH Baugesellschaft \& Co. KG./. Ludger und Ulrike Hofstetter), Slg. 2004, I-3403. Vgl. dazu A. Röthel, Missbräuchliche Klauseln in Verbraucherverträgen - Zur Auslegung des EWGRL 13/93 Art 3 Abs 1 durch den EuGH und durch nationale Gerichte: ZEuP 2005, 421 ff.; Irene Tilmann, Die Auslegung der Richtlinie 93/13/EWG durch den Europäischen Gerichtshof: GPR 2003/04, 182 ff.

13 Vgl. auch E.-M. Kieninger, Die Vollharmonisierung des Rechts der Allgemeinen Geschäftsbedingungen - eine Utopie?, RabelsZ 73 (2009) $792 \mathrm{ff}$.

14 Vgl. zur grundlegenden Konzeption der Schuldrechtsmodernisierung die Beiträge in W. Ernst/R. Zimmermann (Hrsg.), Zivilrechtswissenschaft und Schuldrechtsreform, Tübingen 2001; R. Schulze/H. Schulte-Nölke (Hrsg.), Die Schuldrechtsreform vor dem Hintergrund des Gemeinschaftsrechts, Tübingen 2001, sowie die Dokumentation der Sondertagung "Schuldrechtsmodernisierung" der Zivilrechtslehrervereinigung in JZ 2001, S. 473 ff. (Beiträge von H. Geiger, W.-H. Roth, P. Ulmer, C.-W. Canaris, H.-P. Westermann, H. Roth, D. Leenen). Zur Alternative "große" oder "kleine Lösung", d.h. Umsetzung nur in einem Sondergesetz über den Verbrauchsgüterkauf: B. Dauner-Lieb, Die geplante Schuldrechtsmodernisierung - Durchbruch oder Schnellschuß?, JZ 2001, S. 8 ff.; W. Ernst, EURichtlinie zum Verbrauchsgüterkauf - Optionen für die Umsetzung, in: S. Grundmann/D. Medicus/ W. Rolland (Hrsg.), Europäisches Kaufgewährleistungsrecht, Köln 2000, S. 325 ff.; W. Ernst/B. Gsell, Kaufrechtsrichtlinie und BGB, ZIP 2000, S. $1410 \mathrm{ff}$. 
Frage der Kostenerstattung für den Ausbau einer mangelhaften und Einbau einer mangelfreien Sache, ${ }^{15}$ oder beim Nutzungsersatz im Fall eines Rücktritts, ${ }^{16}$ stellt sich immer wieder die Frage, ob die für den B2C-Bereich vom EuGH gefundenen Lösungen tel quel auch auf den unternehmerischen Bereich, indem keine Bindung an die Richtlinie besteht, übertragbar sind. Im Fall des Nutzungsersatzes hat der deutsche Gesetzgeber bereits reagiert und den vom EuGH geforderten Verzicht auf einen solchen Anspruch auf den Verbrauchsgüterkauf beschränkt (vgl. $\$ 474$ Abs. 2 S. 1 BGB n.F.); ein solches Vorgehen wird auch für die Ein- und Ausbaukosten gefordert. ${ }^{17}$ Das Bestreben des Gesetzgebers der Schuldrechtsmodernisierung, die Einheit der deutschen Zivilrechtskodifikation zu wahren und verbraucherschützende Regeln und Sondergesetze zu (re-) integrieren, wird sich möglicherweise mittel- und langfristig als undurchführbar erweisen. Hans-Werner Micklitz etwa fordert in seinem Gutachten für den 69. Deutschen Juristentag die Wiederausgliederung der Verbraucherschutzvorschriften aus dem BGB und die Schaffung eines eigenen „Verbraucherrechtsgesetzbuches“, ein Weg, den andere Mitgliedstaaten schon länger gehen, siehe den französischen Code de la Consommation und das österreichische Konsumentenschutzgesetz.

Die größtenteils auf das Verbraucherschutzrecht beschränkten Harmonisierungsrichtlinien haben, das hat der vorausgegangene Abschnitt exemplarisch zu zeigen versucht, trotz der entgegengesetzten Bemühungen des Umsetzungsgesetzgebers eine desintegrierende Wirkung auf die nationalen Kodifikationen. ${ }^{18}$ Die Richtlinien lassen sich schwer mit den Idealen der Kodifikation, Systematisierung des juristischen Stoffes und Widerspruchsfreiheit innerhalb des zivilrechtlichen Gesamtsystems, in Einklang bringen. Sie verfolgen konkrete rechtspolitische Ziele, wie die einheitliche Etablierung eines Widerrufsrechts bei Fernabsatz und Haustürgeschäften innerhalb des Gesamtziels der Errichtung und Förderung des europäischen Binnenmarktes. Sie folgen damit ihrer eigenen Logik, die nicht selten quer steht zur Systematik einer Zivilrechtskodifikation, die beispielsweise die Folgen der Ausübung von gesetzlichen und vertraglichen Rücktrittsrechten gemeinsam mit den Folgen eines Widerrufs zu regeln versucht, um eine innere Kohärenz der Rückabwicklungsschuldverhältnisse zu erzielen.

Aber auch dann, wenn man nur die Harmonisierungsrichtlinien selbst ins Auge fasst, stellt sich immer drängender die Frage, ob sie ihr Ziel, die Errichtung und Förderung

15 EuGH 16.6.2011, verb. Rs. C-65/09 und C-87/09, Gebr. Weber GmbH und Ingrid Putz, NJW 2011, S. $2269 \mathrm{ff}$.

16 EuGH 17.4.2008, Rs. C-404/06, Quelle-AG, NJW 2008, S. 1433 ff.

17 S. Lorenz, Ein- und Ausbauverpflichtung des Verkäufers bei der kaufrechtlichen Nacherfüllung - Ein Paukenschlag aus Luxemburg und seine Folgen, NJW 2011, S. 2241 (2244).

18 Vgl. T. Lobinger, Hausgemachte Systemverluste: Die deutsche Schuldrechtsreform und die Verbrauchsgüterkaufrichtlinie, Gemeinschaftsprivatrecht (GPR) 2008, S. $262 \mathrm{ff}$. 
des Binnenmarktes, tatsächlich erreichen können. Das Scheitern der praktischen Implementierung der Klauselrichtlinie zeigt die Grenzen einer sektoriellen Harmonisierung: Der EuGH kann nur sehr begrenzt die Missbräuchlichkeit einer Vertragsklausel beurteilen, wenn der Maßstab der Beurteilung (vgl. $\$ 307$ Abs. 2 Nr. 1 BGB) das weiterhin unvereinheitlichte sonstige Vertragsrecht der Mitgliedstaaten ist. Hier bleibt nur der Rückzug auf allgemeine Floskeln; die konkrete Beurteilung muss dem nationalen Gericht überlassen bleiben. ${ }^{19}$ Allgemeiner gesprochen heißt das, dass das Fehlen eines generellen vertrags- besser noch: vermögensrechtlichen Textes, der einen Bezugsrahmen für die sektorielle Angleichung bieten würde, den Harmonisierungserfolg selbst zu konterkarieren droht.

Eine zweite Gefährdung erwächst aus der Praxis der Mindestharmonisierung: Die verbrauchervertragsrechtlichen Richtlinien wollen die Rechte der Verbraucher im grenzüberschreitenden Verkehr auf ein hinreichend hohes, einheitliches Mindestniveau heben und damit Verbraucher ermutigen, auch grenzüberschreitend Waren und Dienstleistungen zu erwerben. Den Mitgliedstaaten bleibt es dabei gestattet, strengere, für den Verbraucher günstigere Schutzvorschriften beizubehalten oder zu schaffen. Das Instrument der Richtlinie, das immer einen gewissen, wenn auch zunehmend beschränkten Umsetzungsspielraum bietet, lässt überdies im Einzelnen unterschiedlich ausgeformte Regelungen zu. Zusammen mit der Anknüpfung an den gewöhnlichen Aufenthalt des Verbrauchers (Art. 6 Abs. 1 Rom I-Verordnung) ${ }^{20}$ bzw. der Einschränkung der Rechtswahlfreiheit durch den Vorrang der günstigeren Verbraucherschutzvorschriften am gewöhnlichen Aufenthalt des Verbrauchers (Art.6 Abs. 2 Rom I-Verordnung) führt das Prinzip der Mindestharmonisierung aber zu einer Rechtszersplitterung. Unternehmen können gerade kein einheitliches Set von Vertragsbedingungen, Widerrufsbelehrungen und Verbraucherinformationen verwenden, wenn sie mit Verbrauchern in verschiedenen Mitgliedstaaten kontrahieren wollen. In der Praxis hat das (gemeinsam mit anderen Faktoren wie Sprache, Transportkosten, Probleme der Abwicklung von Gewährleistungsfällen und der Rechtsdurchsetzung) bislang zur Folge, dass sich die Verbraucherpreise im Internethandel von Mitgliedstaat zu Mitgliedstaat erheblich unterscheiden; es gibt in der Summe gerade keinen Binnenmarkt für Endverbraucher.

Insgesamt lässt sich nach fast 30 Jahren Praxis sagen, dass das Konzept der Rechtsangleichung jedenfalls im Vertragsrecht an seine Grenzen gestoßen ist. Es ist nicht möglich, ein solches Maß an Rechtseinheit zu schaffen, dass Unternehmen unter Verwendung eines einzigen Vertragsmusters im ganzen Binnenmarkt agieren können, und gleichzeitig die Vorgaben des Art. 288 AEUV ernst zu nehmen und die

19 Vgl. oben, Fn. 12.

20 VO (EG) Nr. 593/2008 über das auf vertragliche Schuldverhältnisse anwendbare Recht („Rom I“), ABl. EG Nr. L 177/6. 
Verbindlichkeit der Richtlinien auf ihre Ziele zu beschränken, damit den Mitgliedstaaten die Freiheit bleibt, die sie brauchen, um die Richtlinienvorgaben in einer Weise umzusetzen, die die Kohärenz ihres jeweiligen Zivilrechtssystems unangetastet lässt. Das jetzige System versucht einen Kompromiss, verfehlt dabei aber letztlich beide Ziele.

\section{Die Idee eines Europäischen Zivilgesetzbuchs - von den Resolutionen des Europäischen Parlaments zum DCFR}

Das Gegenteil eines Kompromisses ist die Idee eines die nationalen Zivilrechtskodifikationen verdrängenden Europäischen Zivilgesetzbuches, das Vertragsrecht, Deliktsrecht und sonstige gesetzliche Schuldverhältnisse sowie Sachenrecht umfasst, das Familien- und Erbrecht aber (zunächst) aussparen würde. Die Vision eines EuZGB ist gewiss älter als die erste Resolution des Europäischen Parlaments von 1989, ${ }^{21}$ aber letztere hat ihr eine erhebliche politische und wissenschaftliche Aufmerksamkeit verschafft. Ein Höhepunkt der dadurch angestoßenen Diskussion war das auf Initiative der niederländischen Ratspräsidentschaft veranstaltete Den Haager Symposium im Jahr 1997.22 Eric Jayme wagte anschließend in seinem Tagungsbericht die Prognose: „Ein ,Euro-ZGB` entspricht dem Geist der Stunde und wird sich kaum aufhalten lassen “. ${ }^{23}$ Seither erscheinen in regelmäßigen Abständen Neuauflagen des von Hartkamp, Hesselink und Hondius herausgegebenen Buchs „Towards a European Civil Code" 24 und eine nicht mehr zu überblickende Fülle an Monographien und Aufsätzen; ${ }^{25}$ auch - teils sehr vehemente - Kritik blieb nicht aus. ${ }^{26} 1999$ formierte sich unter der Leitung von Christian von Bar die Study Group on a European Civil Code, ein Netzwerk von europäischen Rechtswissenschaftlern, die das Ziel verfolgen, "für das Obligationenrecht und Kerngebiete des Sachenrechts einen ko-

21 Vgl. F. Gschnitzer, Geschichte des europäischen Zivilrechts im 19. Und 20. Jahrhundert, entwickelt am Beispiel des österreichischen ABGB, Juristische Blätter (JBl.) 1960, S. 213 (215); J. Basedow, Grundfragen der Vertragsrechtsreform, ZVglRWiss 79 (1980) S. 132 (134); W. Tilmann, Zur Entwicklung eines europäischen Zivilgesetzbuchs, in: W. Jagenburg/G. Maier-Reimer/T. Verhoeven (Hrsg.), Festschrift für Walter Oppenhoff, München 1985, S. 495 ff.; Ernst A. Kramer, Europäische Privatrechtsvereinheitlichung, JBl. 1988, S. 477 ff.

22 Die Referate des Symposiums (Sprecher: E. Hondius, W. van Gerven, W. Tilmann, K. Kerameus, W. Snijders, U. Drobnig, A. Gambaro, M. Bonell, B. Markesinis, O. Lando, U. Mattei, T. Koopmans) sind abgedruckt in European Private Law (ERPL) 1997, S. 455 ff.

23 E. Jayme, Ein Europäisches Zivilgesetzbuch: Die Initiative der Niederlande, IPRax 1997, S. 375.

24 1. Aufl. 1994, aktuell: A. Hartkamp/M. Hesselink/E. Hondius/C. Mak/C. E. du Perron (eds.), Towards a European Civil Code, 4. Aufl. 2010.

25 Vgl. etwa die Nachweise in M. Schmidt-Kessel, Europäisches Zivilgesetzbuch, in: J. Basedow et al. (Hrsg.), Handwörterbuch (Fn. 2), S. 551 ff. sowie die Schrifttumsliste von F.-J. Säcker, Münchener Kommentar BGB, Einl. vor Rdn. 213.

26 Vgl. insbesondere P. Legrand, Against a European Civil Code, Modern Law Review 60 (1997), S. 44 ff.: ders., Antivonbar, Journal of Comparative Law 1 (2005) S. $13 \mathrm{ff}$. 
difizierten Satz von europäischen Rechtsprinzipien zu erarbeiten“. ${ }^{27}$ Die Study Group versteht sich als Nachfolgerin der Lando-Kommission, ${ }^{28}$ unterscheidet sich aber im Zuschnitt der Forschungsgebiete, der über das allgemeine Vertragsrecht hinausgreift und auch spezielle Vertragstypen, gesetzliche Schuldverhältnisse und insbesondere große Teile des Mobiliarsachenrechts einschließt. Parallel arbeitete eine weitere Gruppe (Accademia dei Guisprivatisti Europei) unter der Leitung von Guiseppe Gandolfi an einem Entwurf für ein Europäisches Vertragsgesetzbuch (unter Einschluss der Regeln zum Eigentumsübergang) in der Tradition des französischen Code civil und des italienischen Codice Civile. ${ }^{29}$ Diese Arbeiten haben bisher allerdings kaum Einfluss auf die weitere Entwicklung des Europäischen Privat- bzw. Vertragsrechts gehabt, ob aufgrund der allzu einseitigen Anlehnung an die französische und italienische Tradition zu Recht oder zu Unrecht, soll hier nicht näher thematisiert werden.

Die Kommission griff die Parlamentsinitiative erst (nach erneutem Drängen) ${ }^{30}$ in ihren Mitteilungen von $2001^{31}$ und $2003^{32}$ und nur in Bezug auf das Vertragsrecht auf, legte dabei allerdings einen weiten Vertragsrechtsbegriff zugrunde, der das Bereicherungsrecht und Regeln über die dingliche Wirkung von Mobiliarkreditsicherheiten einschloss. Die erste Mitteilung diskutierte verschiedene Optionen zur weiteren Entwicklung des acquis communautaire, die vom Verzicht auf weitere legislative Maßnahmen bis hin zu der Verabschiedung eines Europäischen Vertragsrechts als

27 Vgl. http://www.sgecc.net/pages/en/introduction/index.introduction.htm: "Our aim is to produce a codified set of Principles of European Law for the law of obligations and core aspects of the law of property” (zuletzt abgerufen am 16.8.2012).

28 Vgl. die von der sogenannten Lando-Kommission (Commission on European Contract Law) erarbeiteten Principles of European Contract Law: O. Lando/H. Beale (eds.), Principles of European Contract Law Parts I and II Combined and revised, The Hague/London/Boston, Kluwer Law International 2000; O. Lando/E. Clive/A. Prüm/R. Zimmermann (eds.), Principles of European Contract Law Part III, The Hague/London/Boston: Kluwer Law International 2003.

29 Eine deutsche Übersetzung des Vorentwurfs ist abgedruckt in ZEuP 2002, S. 139 ff. und S. 365 ff. Vgl. zu dem Projekt G. Gandolfi, Der Vorentwurf eines Europäischen Vertragsgesetzbuchs, ZEuP 2002, S. 1 ff.; H. J. Sonnenberger, Der Entwurf eines Europäischen Vertragsgesetzbuchs der Akademie Europäischer Privatrechtswissenschaftler - ein Meilenstein, RIW 2001, S. 409 ff.

30 Entschließung des Europäischen Parlaments vom 16. März 2000, ABl. EG 2000 Nr. C $377 / 323$ ff.; Entschließung des Europäischen Parlaments vom 15. November 2001, ZEuP 2002, S. 634 ff., dazu Ch. von Bar, Die Resolution des Europäischen Parlaments vom 15. November 2001 zur Annäherung des Zivil- und Handelsrechts der Mitgliedstaaten, ZEuP 2002, S. 629 ff.

31 Mitteilung der Kommission an den Rat und das Europäische Parlament zum Europäischen Vertragsrecht v. 11.7.2001, KOM (2001) 398 endg. Vgl. dazu Ch. von Bar, Die Mitteilung der Europäischen Kommission zum Europäischen Vertragsrecht, ZEuP 2001, S. 799 ff.; S. Leible, Die Mitteilung der Kommission zum Europäischen Vertragsrecht - Startschuss für ein Europäisches Vertragsgesetzbuch?, EWS 2001, S. 471 ff.; D. Staudenmayer, Die Mitteilung der Kommission zum Europäischen Vertragsrecht, EuZW 2001, S. 485 ff.

32 Mitteilung der Kommission an das Europäische Parlament und den Rat: Ein kohärenteres europäisches Vertragsrecht - Ein Aktionsplan, KOM (2003) 68 endgültig. Der Rat hat das Vorgehen der Kommission gebilligt, vgl. Entschließung des Rates zum Thema "Ein kohärenteres europäisches Vertragsrecht", ABl. EU C 2003 C 246/1, abgedruckt auch in ZEuP 2004, S. 424 ff. 
„optionalem Instrument“ reichten. ${ }^{33}$ In der zweiten Mitteilung, dem „Aktionsplan“, wurde deutlich, wohin die Kommission seinerzeit vor allem ${ }^{34}$ tendierte: Das fragmentierte Richtlinienrecht sollte überarbeitet und zusammengefasst werden, um mehr Systematik und Kohärenz zu erreichen. Zu diesem Zweck suchte die Kommission die Hilfe der Wissenschaft, die basierend auf den Principles of European Contract Law, dem acquis communautaire und internationalen Übereinkommen wie dem CISG Modellregeln zum allgemeinen Vertragsrecht sowie Begriffsdefinitionen erarbeiten sollte. Das Ziel war die Schaffung eines Bezugsrahmens für den europäischen Gesetzgeber und den Gerichtshof, damit die Richtlinien künftig nicht mehr im luftleeren Raum schweben würden. Die Idee eines „Gemeinsamen Referenzrahmens" (Common Frame of Reference, CFR) als Werkzeugkasten (tool box) für Legislative und Judikative war geboren.

Die Erarbeitung des wissenschaftlichen Draft Common Frame of Reference (DCFR) wurde zwei Wissenschaftlergruppen anvertraut, neben der bereits erwähnten Study Group on a European Civil Code der Acquis Group, die das Ziel verfolgte, aus dem existierenden, sektoriellen Gemeinschaftsprivatrecht allgemeine Vertragsrechtsprinzipien zu extrahieren bzw. durch Verallgemeinerung von Verbraucherschutzvorschriften zu interpolieren. ${ }^{35}$ Beide Gruppen fanden sich unter dem Dach des „CoPECL Network of Excellence“ zusammen und wurden, mit Forschungsmitteln der Kommission ausgestattet, beauftragt, innerhalb eines abenteuerlich kurzen Zeitraums von nur gut drei Jahren (der Auftrag wurde 2005 vergeben, die Interim Outline Edition erschien bereits im Herbst 2008, ${ }^{36}$ die insgesamt 6563 Druckseiten umfassende Full Edition ${ }^{37}$ ein Jahr später) einen akademischen Entwurf eines Gemeinsamen Referenzrahmens zu erarbeiten - eine Herkulesaufgabe. Zwar lagen die auf breiter rechtsvergleichender Grundlagenforschung basierenden Principles of European Contract Law bereits vor, jedoch bezogen sie zum einen das bereits existierende Gemeinschaftsprivatrecht nicht ein, zum anderen beschränkten sich die Principles auf das allgemeine Vertragsrecht, während der DCFR auch besondere Vertragstypen (Kauf, Dienstleistungsverträge, Miete beweglicher Sachen, Auftrag, Vertriebsverträge, Schenkung und persönliche Sicherheiten) sowie Bücher zum Bereicherungs-, De-

33 KOM (2001) 398 endg., Ziff. 61-69.

34 Als weitere Maßnahmen schlägt sie die Förderung der Ausarbeitung EU-weit verwendbarer Standardvertragsklauseln durch Bereitstellung einer zentralen Webseite und die Formulierung von Leitlinien vor (Fn. 32, Ziff. 81 ff.); dieses Projekt ist mittlerweile eingestellt. Zu der an dritter Stelle vorgeschlagenen Erarbeitung eines optionalen Europäischen Vertragsrechts (aaO., Ziff. 89 ff.) vgl. J. Basedow, Ein optionales Europäisches Vertragsgesetz - opt-in, opt-out, Wozu überhaupt?, ZEuP 2004, S. 1 ff., sowie unten III. 2.

35 Research Group on the Existing EU Private Law (Acquis Group), Principles of the Existing EC Contract Law (Acquis Principles) Contract I, München 2007, Contract II, München 2009.

36 C. von Bar/E. Clive/H. Schulte-Nölke (eds.), Draft Common Frame of Reference, Interim Outline Edition, München 2008.

37 C von Bar/E. Clive (eds.), Principles, Definitions and Model Rules of European Private Law, Draft Common Frame of Reference, Full Edition, München 2009. 
likts-, Mobiliarsachen- und Trust-Recht einschließen sollte. Die Aufgabe wurde zusätzlich dadurch erschwert, dass die Ziele und Verwendungsmöglichkeiten des DCFR von der Kommission nicht hinreichend klar definiert worden waren: Sollte das Werk eine bloße Sammlung von Definitionen und Modellregeln zur Unterstützung bei der Revision der Vertragsrechtsrichtlinien sein? Oder ein wissenschaftliches Restatement eines gemeinsamen Bestands an vertragsrechtlichen Regeln auf der Basis der bereits existierenden Principles of European Contract Law, aber unter Einschluss des acquis communautaire? Sollten die Modellregeln nur ein Referenz- und Ausgangspunkt für praktische und wissenschaftliche Rechtsvergleichung, ohne jede Ambition einer Verwendung für einen verbindlichen europäischen Rechtstext ${ }^{38}$ oder doch eine Blaupause für ein optionales Europäisches Vertrags- oder Vermögensrecht oder gar ein Nukleus für ein künftiges Europäisches Zivilgesetzbuch sein? ${ }^{39}$ Bei allen Fragen (oder „populären Irrtümern“?) ${ }^{40}$ bezüglich der künftigen Verwendungsmöglichkeiten ließen die äußere Gestalt, der Zuschnitt und Aufbau der Bücher und Kapitel und nicht zuletzt der Gehalt der Vorschriften keinerlei Zweifel an dem eigentlichen Traum der Verfasser: „The DCFR is a European civil code, in all but na$m e$ ". ${ }^{41}$

\section{Vom DCFR über die Horizontalrichtlinie zum optionalen europäischen Kaufrecht oder: „Parturient montes, nascetur ridiculus mus“ ${ }^{\prime 42}$}

Heute, nur drei Jahre nach der Veröffentlichung der Gesamtausgabe des DCFR ist die wissenschaftliche Aufmerksamkeit für das Werk ${ }^{43}$ bereits wieder am Abflauen; die Aufregung hat sich gelegt, Vorhaben wie die Erstellung eines Großkommentars zum DCFR analog den Kommentierungen des BGB liegen auf Eis. Die rechtspoliti-

38 So H. Schulte-Nölke, Arbeiten an einem europäischen Vertragsrecht - Fakten und populäre Irrtümer, NJW 2009, S. 2161 ff.; Schulte-Nölke ist einer der Mitherausgeber des DCFR.

39 So die Perzeption des Projekts in der veröffentlichen Meinung, vgl. J. Jahn, Rettet das BGB vor Brüssel, FAZ v. 17.10.2006: „Eifrige Doktoranden basteln am Reißbrett ein europäisches

Zivilgesetzbuch, das niemand braucht. Weitgehend unbeachtet, aber wild entschlossen arbeitet eine kleine Gruppe von Rechtswissenschaftlern am Abschied vom Bürgerlichen Gesetzbuch (BGB) und von anderen nationalen Paragraphenwerken. Die Europäische Kommission in Brüssel hat nämlich mit Unterstützung des Europaparlaments - den Auftrag erteilt, einen "Gemeinsamen Referenzrahmen" für das Vertragsrecht der Mitgliedstaaten auszuarbeiten. Was hinter diesem harmlos klingenden Namen steckt, ist dasselbe wie das, was Europapolitiker und Eurokraten sonst hinter wohltönenden Floskeln wie Harmonisierung, Kohärenz und Konvergenz verstecken: die Vereinheitlichung gewachsener Strukturen zugunsten einer zentralistischen Vorgabe. ".

40 Vgl. Fn. 38.

41 M. Hesselink, The Common Frame of Reference as a Source of European Private Law, Tulane LR 83 (2009), S. 919 ff. Hesselink war in leitender Funktion an der Erarbeitung des DCFR beteiligt. Vgl. auch N. Jansen/R. Zimmermann, Was ist und wozu der DCFR?, NJW 2009, S. 3401 (3402) m.w.N.

42 Vgl. Horaz, De arte poetica, Vers 139; vgl. auch S. Leible, Von einem Berg sowie Mäusen und anderen Tieren - Das optionale Instrument ante portas, EuZW 2011, S. 809 f.

43 Vgl. aus der umfangreichen Literatur nur H. Eidenmüller/F. Faust/H. Grigoleit/N. Jansen/G. Wagner/ R. Zimmermann, Der Gemeinsame Referenzrahmen für das Europäische Privatrecht, JZ 2008, S. 529 ff.; M. Schmidt-Kessel (Hrsg.), Der gemeinsame Referenzrahmen, München 2009. 
sche Entwicklung ist, einstweilen jedenfalls, über den DCFR hinweggegangen. ${ }^{44}$ Das erste der von der Kommission seit Erscheinen der Interim Outline Edition 2008 vorangetriebenen Projekte, die Horizontalrichtlinie, nimmt praktisch keinen Bezug auf den DCFR, beim zweiten Projekt, dem Gemeinsamen Europäischen Kaufrecht (Common European Sales Law - CESL) sind inhaltliche Einflüsse zwar unverkennbar, aber das CESL unterscheidet sich in Zielsetzung, Zuschnitt und den Detailregelungen markant von einem zunächst anvisierten „politischen“ CFR:

\section{Verbraucherrechterichtlinie}

Die Verfasser des DCFR wurden von der Kommission unter anderem deshalb unter so massiven Zeitdruck gesetzt, weil das im Aktionsplan von 2003 an erster Stelle figurierende Projekt einer Zusammenfassung der verbrauchervertragsrechtsangleichenden Richtlinien in einer Horizontalrichtlinie möglichst rasch verwirklicht werden sollte. Der Vorschlag der Kommission, ${ }^{45}$ der am 8.10.2008, also fast zeitgleich mit der Interim Outline Edition des DCFR, veröffentlicht wurde, vereinte vier existierende Richtlinien, die Fernabsatzrichtlinie, die Haustürwiderrufsrichtlinie, die Klauselrichtlinie und die Verbrauchsgüterkaufrichtlinie ${ }^{46}$ zu einer Gesamtverbraucherrechterichtlinie. Die Zusammenfügung sollte allerdings nicht nur Unstimmigkeiten, wie etwa die unterschiedliche Ausgestaltung der Widerrufsrechte, beseitigen, sondern vor allem statt der bisherigen Mindestharmonisierung eine Vollharmonisierung herbeiführen (vgl. Art. 4 des Vorschlags), damit die Unternehmen ihre grenzüberschreitenden Verbraucherverträge nicht länger an den unterschiedlichen nationalen Umsetzungsvorschriften mit ihren divergierenden Verbraucherschutzniveaus ausrichten müssten. Dies scheiterte indes, jedenfalls im Hinblick auf die beiden für das Vertragsrecht zentralen Richtlinien, die Klauselrichtlinie und die Verbrauchsgüterkaufrichtlinie, am Widerstand der Mitgliedstaaten. Das Ergebnis ist eine Verbraucherrechterichtlinie, ${ }^{47}$ die nur noch die Haustürwiderrufs- und die Fernabsatzrichtlinie zusammenfasst, sich weitgehend in einem paste and copy erschöpft und das Prinzip der Vollharmonisierung zwar noch vollmundig in Art. 4 verkündet, in den Einzelvorschriften aber teilweise wieder zurücknimmt (vgl. etwa Art. 5 Abs. 4, Art. 6 Abs. 7). Die Kommission nennt dies euphemistisch „targeted harmonisation" ${ }^{48}$ Norbert Reich spricht treffender von „Halbharmonisierung “. ${ }^{49}$ Überdies war selbst im ursprünglichen Kommissionsvorschlag kaum eine Spur des DCFR zu erkennen. Nicht einmal die Begriffsbestimmungen in Art. 2 des Kommissionsvor-

\footnotetext{
44 Vgl. P. Mankowski, CESL - Who needs it?, IHR 2012, S. 45 ff., der den DCFR als "politisch gescheitert" betrachtet.

$45 \operatorname{KOM}(2008) 614$ endgültig.

46 Siehe Nachweise Fn. 6 ff.

47 RL 2011/83/EU vom 25.10.2011 über die Rechte der Verbraucher, ABl. Nr. L 304/64.

$48 \mathrm{http} / / / \mathrm{www}$.europolitics.info/commission-now-considering-targeted-harmonisation-artb264092-10.html (zuletzt abgerufen am 16.8.2012).

49 N. Reich, Von der Minimal- zur Voll- zur Halbharmonisierung, ZEuP 2010, S. 7.
} 
schlags stimmten mit den Definitionen im Annex des DCFR überein. Der gerade neu eingerichtete Werkzeugkasten (tool box) der Kommission blieb dort, wo er auch im praktischen Leben akademischer Haushalte bedauerlicherweise meist verbleibt: Im Schrank. ${ }^{50}$

\section{Der Kommissionsvorschlag für ein gemeinsames Europäisches Kaufrecht}

Etwas stärkeren inhaltlichen Einfluss nahm der DCFR auf ein zweites Vorhaben, das die Kommission nach dem Scheitern des Vollharmonisierungsprojekts mit Verve vorantrieb, der Erarbeitung eines optionalen Europäischen Kaufrechts. Zunächst einmal schien es, als wolle die Kommission tatsächlich einen „politischen“ CFR in mehr oder weniger weitgehender Anlehnung an den DCFR formulieren, wobei aber unklar blieb, welche rechtliche Form (unverbindliche Empfehlung, interinstitutionelle Vereinbarung) ein solcher Text haben könnte. Die im April 2010 eingesetzte Expertengruppe erhielt den Auftrag

„diejenigen Teile des DCFR [zu] identifizieren, die unmittelbar oder mittelbar für das Vertragsrecht von Bedeutung sind, und die Regeln gegebenenfalls [zu] restrukturieren und [zu] überarbeiten". 51

Indes wurde spätestens mit der Veröffentlichung der „Machbarkeitsstudie“ durch die Expertengruppe im Mai 2011 (eine überarbeitete Version erschien im August $2011)^{52}$ deutlich, dass die Kommission längst auf ein optionales europäisches Kaufrecht zusteuerte, also nur einen kleinen Ausschnitt aus den DCFR-Arbeiten auswählen und in die Form eines optionalen Instruments gießen würde. Diese Entscheidung dürfte bereits getroffen gewesen sein, als die Kommission in dem im Juli 2010 nachgeschobenen Grünbuch zum Europäischen Vertragsrecht noch einmal sieben Optionen zur weiteren Entwicklung zur Diskussion stellte, von der bloßen Veröffentlichung der Machbarkeitsstudie bis zu einer Verordnung über ein Europäisches Zivilgesetzbuch. ${ }^{53}$ Daran, dass die Konsultationsfrist bis zum Januar 2011 lief, der Text der Expertengruppe für ein Europäisches Kaufrecht, der in weiten Teilen dem endgültigen Text des Kommissionsvorschlags entspricht, ${ }^{54}$ aber bereits im Mai 2011 vorlag, ist abzulesen, dass es sich bei der durch das Grünbuch eingeleiteten Konsultation um ein bloßes Feigenblatt handelte. ${ }^{55}$ Den vorläufigen Schlusspunkt bildet der im Oktober 2011 veröffentlichte Vorschlag der Kommission für eine Verordnung

50 Vgl. Ch. Wendehorst, The CFR and the Review of the Acquis Communautaire, in: M. Schmidt-Kessel (Hrsg.), Der gemeinsame Referenzrahmen, München 2009, S. 323 ff.

51 ABl. L 105/109 vom 27.4.2010.

52 http://ec.europa.eu/justice/contract/files/feasibility-study_en.pdf (zuletzt abgerufen am 16.8.2012).

53 KOM (2010) 348 endgültig.

54 Vgl. die in H. Schulte-Nölke/F. Zoll/N. Jansen/R. Schulze (Hrsg.), Der Entwurf für ein optionales europäisches Kaufrecht, München 2012, S. 297 ff. abgedruckte Synopse.

55 H. Eidenmüller/N. Jansen/E.-M. Kieninger/G. Wagner/R. Zimmermann, Der Vorschlag für eine Verordnung über ein Gemeinsames Europäisches Kaufrecht, JZ 2012, S. 269 (271). 
über ein Gemeinsames Europäisches Kaufrecht, aufgeteilt in einen materiellrechtlichen Annex und eine vorgeschaltete Anwendungsverordnung.

Vom DCFR oder der Idee eines politischen CFR unterscheidet sich der CESL-Vorschlag vor allem durch seinen äußerst begrenzten Zuschnitt. Keines der nicht-vertragsrechtlichen Bücher (Bereicherungsrecht, Geschäftsführung ohne Auftrag, Deliktsrecht, Sachenrecht, Trust) findet sich in dem Vorschlag wieder. Selbst die Frage des Eigentumsübergangs, immerhin ein zentraler Aspekt bei der Erfüllung eines jeden Kaufvertrags, bleibt ausgespart. Sachlich enthält der Kommissionstext - wie das CISG - im Wesentlichen nur Kaufrecht, allerdings einschließlich der verbraucherschützenden Informationspflichten und Widerrufsrechte und ergänzt um bestimmte Teile des allgemeinen Vertragsrechts, namentlich Vorschriften über die Anfechtung wegen Willensmängeln, über die Einbeziehungs- und Inhaltskontrolle vorformulierter Vertragsbestimmungen und über die Verjährung. Einbezogen in den sachlichen Anwendungsbereich sind weiterhin Verträge über digitale Inhalte und Dienstleistungen, die mit Kaufverträgen in Verbindung stehen, wie die Installation und Wartung der Kaufsache. Im Übrigen aber finden sich im „optionalen Instrument“ keine Vorschriften über Dienstleistungsverträge wie Auftrag, Vertriebsverträge, Behandlungsverträge etc., die im DCFR mit teilweise durchaus innovativen Regelungen vertreten waren. Auch viele Gegenstände des allgemeinen Vertrags- bzw. Schuldrechts, wie beispielsweise die Stellvertretung, die Abtretung und die Mehrheit von Schuldnern und Gläubigern bleiben ausgespart und damit trotz Wahl eines künftigen CESL dem unvereinheitlichten, nach Maßgabe des Kollisionsrechts zu bestimmenden nationalen Recht unterstellt. Räumlich-persönlich beschränkt sich der Anwendungsbereich, wenn man von den mitgliedstaatlichen Erweiterungsmöglichkeiten (Art. 13 CESL-Reg.) absieht, auf grenzüberschreitende Verträge zwischen Unternehmen und Verbrauchern (B2C) und zwischen Unternehmen (B2B), wenn mindestens eines ein „kleines und mittleres Unternehmen“ (KMU) im Sinn der Definition in Art.7 Abs. 2 CESL-Reg. ist.

Die Kommission hat sich durch diesen Zuschnitt von der Idee eines politischen Referenzrahmens für die weitere Entwicklung des Europäischen Privatrechts abgewendet, ja man kann nicht einmal von einem optionalen Europäischen Vertragsrecht sprechen. ${ }^{56}$ Es handelt sich bei dem Vorschlag im Wesentlichen um ein wählbares (opt-in) Einheitsrecht, das auf grenzüberschreitende Internetkaufverträge zwischen Unternehmen und Verbrauchern zugeschnitten ist, und das sich inhaltlich vor allem an den Verbrauchervertragsrichtlinien und dem CISG orientiert. Das optionale Instrument präsentiert sich als ein Ersatz für die gescheiterte Vollharmonisierung: Da es nicht gelungen ist, Unternehmen ein vollständig harmonisiertes mitgliedstaatliches 
Verbrauchervertragsrecht bereit zu stellen, sollen sie auf diese Weise zumindest die Option haben, mittels des berühmten „blue button" 57 freiwillig auf der Basis eines Einheitstextes grenzüberschreitend mit Verbrauchern bzw. KMU Kaufverträge zu schließen. Ob es der zuständigen Kommissarin, Viviane Reding, gelingen wird, trotz der Subsidiaritätsrüge, die etwa der Deutsche Bundestag erhoben hat, ${ }^{58}$ trotz der berechtigten Zweifel an der Richtigkeit der gewählten Kompetenzgrundlage (Art. 114 AEUV anstelle von Art. 352 AEUV) ${ }^{59}$ und trotz der teils massiven Kritik, die sowohl an dem Verfahren der Entstehung ${ }^{60}$ als auch am Inhalt des CESL ${ }^{61}$ geäußert worden ist, das Projekt zu einem erfolgreichen Abschluss zu bringen, darf bezweifelt werden. Noch zweifelhafter ist, ob der Text, sollte er mit dem vorgeschlagenen Inhalt verabschiedet werden, in der Praxis gewählt werden wird. ${ }^{62}$ Unabhängig davon ist für unser Thema indes festzustellen, dass die Vision einer Europäischen Zivilrechts- oder auch nur Schuld- oder Vertragsrechtskodifikation jedenfalls derzeit politisch nicht auf der Agenda steht. Selbst beschränkt auf das Kaufrecht kann in der Diktion Zenati-Castaings allenfalls von einer europäischen Kaufrechtskompilation die Rede sein, in Ermangelung eines systematisch und inhaltlich in sich stimmigen Texts jedoch nicht von einer Kaufrechtskodifikation. ${ }^{63}$

57 Vgl. H. Schulte-Nölke, Der Blue Button kommt, ZEuP 2011, S. 749 ff.; vgl. aber auch G. Wagner, Der Blue Button klemmt, ZEuP 2012, S. 455 ff.

58 BT-Drucks. 17/8000.

59 Vgl. H. Eidenmüller/N. Jansen/E.-M. Kieninger/G. Wagner/R. Zimmermann, Vorschlag (Fn. 55), JZ 2012, S. 269 (274); P.-Ch. Müller-Graff, Ein fakultatives europäisches Kaufrecht als Instrument der Marktordnung?, in: H. Schulte-Nölke et al. (Hrsg.) (Fn. 54), S. 21 (35); J. Basedow, Fakultatives Unionsprivatrecht oder: Grundlagen des 28. Modells, in: D. Joost/H. Oetker/M. Paschke (Hrsg.), Festschrift für Franz-Jürgen Säcker, München 2011, S. 29 (38 ff.); ders., Art. 114 AEUV als Rechtsgrundlage eines optionalen EU-Kaufrechts: Eine List der Kommission?, EuZW 1/2012, Editorial; Max Planck Institute for Comparative and International Private Law, Policy Options for Progress Towards a European Contract Law, RabelsZ 75 (2011) S. 371 ff., Rdn. 41 ff., 54; J.-J. Kuipers, The Legal Basis of a European Optional Instrument, ERPL 2011, S. 545 (559); a. A. (pro Art. 114 AEUV) N. Reich/ H.-W. Micklitz, Wie „optional“ ist ein „optionales“ EU-Vertragsrecht?, EWS 2011, S. 113 (114); Stellungnahme der Bundesrechtsanwaltskammer, IHR 2012, S. 53 (59). Dagegen verneint H. Grigoleit, Der Entwurf für ein Gemeinsames Europäisches Kaufrecht: Funktionsbedingungen, EU-Kompetenz und Perspektiven, in: O. Remien/S. Herrler/P. Limmer (Hrsg.), Gemeinsames Europäisches Kaufrecht für die EU?, München 2012, S. 67 (75 ff.) sowohl eine auf Art. 114 als auch eine auf Art. 352 AEUV gestützte Kompetenz.

60 Vgl. W. Doralt, Rote Karte oder grünes Licht für den Blue Button?, AcP 211 (2011), S. 1 ff.

61 Vgl. H. Eidenmüller/N. Jansen/E.-M. Kieninger/G. Wagner/R. Zimmermann, Vorschlag (Fn. 55), JZ 2012, S. 269 ff. und die überwiegend kritischen Beiträge in O. Remien/S. Herrler/P. Limmer (Hrsg.) (Fn. 59); S. Lorenz, Vortrag Sondertagung Zivilrechtslehrervereinigung April 2012 Bonn, AcP 212 (2012) (im Erscheinen); N. Jansen, ZEuP 2012 (im Erscheinen); S. Balthasar, Das Gemeinsame Europäische Kaufrecht - eine Analyse aus unternehmerischer Sicht, RIW 2012, S. 361 ff.; R. Zimmermann, Perspektiven des künftigen österreichischen und europäischen Zivilrechts, JBl. 2012, S. 2 ff. A.A. F. Graf von Westphalen, Eine wohl unwiederbringliche Chance, EWS 7/2011, Die erste Seite; ders., Das optionale Europäische Kaufrecht - eine Chance für Verbraucher und Unternehmer?, ZIP 2011, S. $1985 \mathrm{ff}$.

62 Eine Hürde sind insbesondere der nicht weit genug gespannte räumlich-persönliche Anwendungsbereich sowie die besonderen Voraussetzungen für die Wahl des CESL in Verbraucherverträgen, vgl. $H$. Eidenmüller/N. Jansen/E.-M. Kieninger/G. Wagner/R. Zimmermann, Vorschlag (Fn. 55), S.269 (275f.).

63 F. Zenati-Castaing, L'avenir de la codification (Fn. 1), S. 359. 


\section{B. Vom Römer Übereinkommen 1980 zur Idee einer Europäischen IPR-Kodifikation}

\section{Die Ablösung der Staatsverträge durch die „Rom“- und „Brüssel“-Verordnungen}

Anders als die Geschichte der Idee eines Europäischen Zivilgesetzbuchs begann die Entwicklung des Europäischen Internationalen Privatrechts unspektakulär mit dem Römer Übereinkommen von 1980 über das auf Schuldverträge anzuwendende Recht,${ }^{64}$ einem Rechtsakt, der in Ermangelung einer eigenen Kompetenz der EU für die Vereinheitlichung des Kollisionsrechts ganz in der Tradition der kollisionsrechtsvereinheitlichenden Staatsverträge stand. Die binnenmarktfinalen Kollisionsnormen, die dem Schutz der durch die Harmonisierungsrichtlinien erreichten Angleichung gegenüber der Wahl eines drittstaatlichen Rechts dienen sollten, fanden sich nicht im Römer Übereinkommen, sondern in verschiedenen Verbraucherrechtsrichtlinien, die dementsprechend zunächst in den verbraucherrechtlichen Sondergesetzen, ab der Fernabsatzrichtlinie dann gesammelt im EGBGB (früher Art.29a, jetzt Art.46b EGBGB) in nationales Recht umgesetzt wurden. ${ }^{65}$

Versteht man den Begriff des Internationalen Privatrechts umfassend, so wie das etwa in Frankreich der Fall ist, so muss der Beginn bereits mit einem früheren Staatsvertrag angesetzt werden, dem Brüsseler Übereinkommen von 1968 über die internationale Zuständigkeit, die Anerkennung und Vollstreckung von gerichtlichen Entscheidungen in Zivil- und Handelssachen zwischen den sechs Gründungsmitgliedstaaten der EWG. ${ }^{66}$ Beide Übereinkommen unterscheiden sich nicht nur in der rechtlichen Form von den ersten materiell-rechtlichen Richtlinien, sie schufen auch von Anfang an Einheitsrecht, nicht nur angeglichenes Recht. Alles andere wäre unpraktikabel gewesen, denn das Ziel der Vereinheitlichung des Kollisionsrechts liegt darin, dass das anwendbare Recht unabhängig von der internationalen Zuständigkeit von allen mitgliedstaatlichen Gerichten in gleicher Weise bestimmt wird. Umsetzungsspielräume und Mindestharmonisierung wären hier fehl am Platz. Erleichtert wurde die europäische Vereinheitlichung des IPR und IZVR dadurch, dass anders als bei der Angleichung des materiellen Rechts durch Richtlinien kein Neuland betreten werden musste, sondern auf die jahrzehntelange Praxis der Kollisionsrechtsvereinheitlichung

64 Europäisches Übereinkommen über das auf vertragliche Schuldverhältnisse anzuwendende Recht vom 19. Juni 1980, ABl. EG 1980 Nr. L 266/1.

65 Zur Frage der Sinnhaftigkeit ihrer Beibehaltung neben dem erweiterten Art. 6 Rom I-VO und dem neu eingeführten Art. 3 Abs. 4 Rom I-VO vgl. E.-M. Kieninger, Der grenzüberschreitende Verbrauchervertrag zwischen Richtlinienkollisionsrecht und Rom I-Verordnung, in: D. Baetge/J. von Hein/M. von Hinden (Hrsg.), Die richtige Ordnung, Festschrift für Jan Kropholler, Tübingen 2008, S. 499 ff.; S. Leible, Brauchen wir noch Art. 46 b EGBGB?, in: H. Kronke/K. Thorn (Hrsg.), Grenzen überwinden - Prinzipien bewahren, Festschrift für Bernd von Hoffmann, Bielefeld 2011, S. $230 \mathrm{ff}$.

66 Brüsseler EWG-Übereinkommen über die gerichtliche Zuständigkeit und Vollstreckung gerichtlicher Entscheidungen in Zivil- und Handelssachen vom 27.9.1968 in der Fassung des 4. Beitrittsübereinkommens, BGBl. 1998 II 1412. 
im Rahmen der Haager Konferenz für Internationales Privatrecht ${ }^{67}$ zurückgegriffen werden konnte.

Der am 1.5.1999 in Kraft getretene Amsterdamer Vertrag markiert den wohl wichtigsten Einschnitt in der Entwicklung des europäischen IPR und IZVR: ${ }^{68}$ Noch während Verhandlungen über weitere Staatsverträge, etwa das Europäische Insolvenzübereinkommen und das Übereinkommen über das auf außervertragliche Schuldverhältnisse anwendbare Recht („Rom II“-Übereinkommen) andauerten, erhielt die EG eine originäre Kompetenz zur Vereinheitlichung des Internationalen Privat- und Verfahrensrechts (vgl. Art. 61 lit c i.V.m. Art. 65 lit b EGV i.d.F. des Amsterdamer Vertrags, jetzt Art. 81 Abs. 2 lit c AEUV), von der sie in der Folge regen Gebrauch gemacht hat. Mit Ausnahme von Maßnahmen auf dem Gebiet des Familienrechts, für die Einstimmigkeit im Rat erforderlich ist (vgl. Art. 67 Abs. 5 2. Sp.strich EGV bzw. Art. 81 Abs. 3 AEUV), gilt für die hierauf gestützten Verordnungen das ordentliche Gesetzgebungsverfahren, so dass eine qualifizierte Mehrheit im Rat genügt. ${ }^{69}$ Der Lissabonner Vertrag hat die Kompetenz der EU zusätzlich ausgeweitet: Sie besteht jetzt unabhängig davon, ob die Vereinheitlichungsmaßnahme für das Funktionieren des Binnenmarkts erforderlich ist; das letztgenannte Kriterium ist in Art. 81 Abs. 2 AEUV zu einem bloßen Regelbeispiel herabgesunken, ${ }^{70}$ was insbesondere Maßnahmen im Bereich des Personenrechts (Erbrecht, Familienrecht, Unterhaltsrecht) erleichtert. Der argumentative Aufwand, den man treiben musste, um den Binnenmarktbezug aus der Personenfreizügigkeit abzuleiten, ${ }^{71}$ kann nunmehr entfallen. Zweifel an der Zuständigkeit der EU zur Vereinheitlichung des IPR und des IZVR gibt es - anders als im materiellen Privatrecht ${ }^{72}$ - in Anbetracht der klaren Kompetenznorm nicht. Allenfalls in Bezug auf die Reichweite der Vereinheitlichungsinstrumente wurde die Kompetenz diskutiert, etwa bei der Frage, ob sich die internationale Zuständigkeit auch dann nach der EuGVVO (bzw. zuvor nach dem Brüsseler Übereinkommen) richtet, wenn der Sachverhalt nur Bezüge zu einem Mit-

67 Vgl. J. Pirrung, Haager Konferenz für Internationales Privatrecht, in: J. Basedow et al. (Hrsg.), Handwörterbuch (Fn. 2), S. 793 ff.

68 Vgl. zum Folgenden E. Jayme/Ch. Kohler, Europäisches Kollisionsrecht 1997 - Vergemeinschaftung durch Säulenwechsel?, IPRax 1997, S. 385 ff.; dies., Europäisches Kollisionsrecht 1999 - Die Abendstunde der Staatsverträge, IPRax 1999, S. $401 \mathrm{ff.}$

69 Näher R. Wagner, Praktische Fragen mit der Rechtsvereinheitlichung in der justiziellen Zusammenarbeit in Zivilsachen, in: E.-M. Kieninger/O. Remien (Hrsg.), Europäische Kollisionsrechtsvereinheitlichung, Baden-Baden 2012, S. $51 \mathrm{ff}$.

70 Vgl. E. Jayme, Das europäische IPR löst sich vom Binnenmarkt, IPRax 2008, S. 72 f.; A. Dutta, Europäische Integration und nationales Privatrecht nach dem Vertrag von Lissabon: die Rolle des Internationalen Privatrechts, EuZW 2010, S. 530 (531) m.w.N.

71 Vgl. etwa noch R. Wagner, EG-Kompetenz für das Internationale Privatrecht in Ehesachen?, RabelsZ 68 (2004), S. $119 \mathrm{ff}$.

72 Vgl. H. Grigoleit, in: O. Remien/S. Herrler/P. Limmer (Hrsg.) Fn. 59. 
gliedstaat aufweist, im Übrigen aber nur mit Drittstaaten verbunden ist. ${ }^{73}$ Für die Praxis setzte der EuGH der Diskussion ein Ende, ${ }^{74}$ mit der Aufgabe der strikten Binnenmarktfinalität in Art. 81 Abs. 2 AEUV ist ihr gänzlich der Boden entzogen.

Allerdings ist die Kompetenz der EU mit einem schwerwiegenden Geburtsfehler behaftet: ${ }^{75}$ Die justizielle Zusammenarbeit wurde gemeinsam mit den Politikbereichen Asyl, Einwanderung und Visaangelegenheiten in Titel IV des Amsterdamer Vertrags eingestellt, mit der Folge, dass Dänemark einen Vorbehalt gegenüber dem gesamten Titel erklärt hat und an keinem der hierauf gestützten Gesetzgebungsverfahren teilnimmt. Das alleine ließe sich noch verschmerzen, denn die Praxis zeigt, dass Dänemark letztlich gezwungen ist, die neuen Verordnungen per Staatsvertrag mit der EU zu übernehmen, ohne zuvor an den Verhandlungen der Verordnungen beteiligt gewesen zu sein. ${ }^{76}$ Größere Probleme schaffen die Sonderrollen des Vereinigten Königreichs und Irlands: Beide Staaten haben die Möglichkeit, jeweils in Bezug auf einzelne Verordnungen ein opt-in zu erklären, was auch noch nach Abschluss der Verhandlungen und Annahme der Verordnung möglich ist. Von dem hierdurch gewährten Erpressungspotenzial ${ }^{77}$ hat das Vereinigte Königreich bereits intensiven Gebrauch gemacht, etwa indem es gedroht hat, die Rom I-Verordnung, die Nachfolgerin des Römer Übereinkommens, nicht anzunehmen, wenn die Regelung über die Berücksichtigung ausländischer Eingriffsnormen (Art. 9 Abs. 3 Rom I-VO) nicht in seinem Sinne ausfallen würde.

Daran, dass ganze Abschnitte des EGBGB bereits aufgehoben sind und weitere in Kürze folgen werden, lässt sich ablesen, wie weit die Vergemeinschaftung des Kollisionsrechts bereits vorangeschritten ist: ${ }^{78}$ Das internationale Vertragsrecht ist in der Rom I-VO enthalten, das auf außervertragliche Schuldverhältnisse (Delikts-, Bereicherungsrecht und Geschäftsführung ohne Auftrag) anwendbare Recht richtet sich nach der Rom II-VO. ${ }^{79}$ Die jüngst (am 21. Juni 2012) in Kraft getretene Rom III-

73 Vgl. J. Kropholler, Europäisches Zivilprozessrecht, 8. Aufl. 2005, Frankfurt a.M., vor Art. 2 EuGVVO Rdn. 8.

74 EuGH 1.3.2005, Rs. C-281/02, Owusu/Jackson, Slg. 2005 I 1383. Siehe auch Erwägungsgrund (8) zur EuGVVO, der nur Bezüge zu einem Mitgliedstaat verlangt.

75 Vgl. zum Folgenden R. Wagner, in: E.-M. Kieninger/O. Remien (Hrsg.) (Fn. 69), S. 51 (64 ff.).

76 Siehe die Übernahme der EuGVVO durch Staatsvertrag v. 9.10.2005 zwischen der EG und Dänemark, ABl. EU Nr. L 299, S. 62.

77 So die Formulierung von R. Wagner, in: E.-M. Kieninger/O. Remien (Hrsg.) (Fn. 69), S. 51 (65).

78 Vgl. D. Henrich, Europäisierung des internationalen Familienrechts: Was bleibt vom EGBGB?, J. Bernreuther/R. Freitag/S. Leible et al. (Hrsg.), Festschrift für Ulrich Spellenberg, München 2010, S. $195 \mathrm{ff}$.

79 Dass Art. 40-42 EGBGB noch nicht gestrichen sind, liegt daran, dass die Rom II-Verordnung einige Bereiche aus ihrem sachlichen Anwendungsbereich ausnimmt, insbesondere das auf Persönlichkeitsrechtsverletzungen anwendbare Recht. 
VO, ${ }^{80}$ die das auf Ehescheidungen anwendbare Recht regelt, hat Art. 17 Abs. 1 und 2 EGBGB bereits verdrängt, auch wenn das entsprechende Bundesgesetz noch auf sich warten lässt. Die letztgenannte Verordnung konnte allerdings nur im Rahmen einer verstärkten Zusammenarbeit verabschiedet werden und gilt daher lediglich in 15 von 27 Mitgliedstaaten (darunter in Deutschland), da im Rat die in Familiensachen erforderliche Einstimmigkeit nicht zu erzielen war. Die Europäische Erbrechtsverordnung ${ }^{81}$ (an der sich das UK und Irland nicht beteiligen werden) ist im Juni 2012 verabschiedet worden und wird in ihren wesentlichen Teilen ab dem 17.8.2015 gelten. Eine Verordnung zum internationalen Ehegüterrecht ist in Vorbereitung. ${ }^{82}$ Die seit Mitte 2011 anwendbare Europäische Unterhaltsverordnung ${ }^{83}$ stellt kollisionsrechtlich insoweit eine Besonderheit dar, als sie in Art. 15 auf das Haager Unterhaltsprotokoll 2007 verweist, so dass wenigstens in diesem Bereich der dringend notwendige Gleichklang zwischen europäischer und (potenziell) weltweiter Kollisionsrechtsvereinheitlichung erzielt wurde, denn Unterhaltsschuldner setzen sich nicht nur in andere EU-Mitgliedstaaten ab, auch wenn die Freizügigkeit dies natürlich besonders erleichtert.

Eng verzahnt mit dem Kollisionsrecht ist die Bestimmung der internationalen $\mathrm{Zu}$ ständigkeit: Nur wenn ein mitgliedstaatliches Gericht entscheidet, wendet es das vergemeinschaftete Kollisionsrecht an. Überdies hängt die Verwirklichung des Binnenmarktes, was trotz der Herabstufung zu einem Regelbeispiel in Art. 81 AEUV immer noch das wichtigste Ziel der europäischen Gesetzgebungstätigkeit auch im Bereich des IPR und IZVR ist, noch mehr von der zügigen und problemlosen Anerkennung und Vollstreckung von Urteilen aus anderen Mitgliedstaaten und der möglichst sicheren Bestimmung der Gerichtszuständigkeit ab als von der einheitlichen Beantwortung der Frage nach dem anwendbaren Recht. Dementsprechend erstreckt sich die Gesetzgebungstätigkeit der EU ebenso auf die genannten Bereiche des Internationalen Zivilverfahrensrechts, siehe die in Anlehnung an das Brüsseler Überein-

80 Verordnung (EU) Nr. 1259/2010 des Rates vom 20.12.2010 zur Durchführung einer Verstärkten Zusammenarbeit im Bereich des auf die Ehescheidung und Trennung ohne Auflösung des Ehebandes anzuwendenden Rechts, ABl. EU 2010 L 343/10.

81 Verordnung (EU) Nr. 650/2012 vom 4. Juli 2012 über die Zuständigkeit, das anzuwendende Recht, die Anerkennung und Vollstreckung von Entscheidungen und die Annahme und Vollstreckung öffentlicher Urkunden in Erbsachen sowie zur Einführung eines Europäischen Nachlasszeugnisses, ABL. EU L 201/107.

82 Vorschlag für eine Verordnung des Rates über die Zuständigkeit, das anzuwendende Recht, die Anerkennung und die Vollstreckung von Entscheidungen im Bereich des Ehegüterrechts, KOM (2011) 126.

83 Verordnung (EG) Nr. 4/2009 über die Zuständigkeit und das anwendbare Recht, die Anerkennung und Vollstreckung von Entscheidungen und die Zusammenarbeit in Unterhaltssachen (EuUntVO) v. 18.12.2008, ABl. EU 2009 Nr. L 7/1, anwendbar ab dem 18. Juni 2011, vgl. Art. 76 Abs. 3 EuUntVO. 
kommen von 1968 Brüssel I (EuGVVO) ${ }^{84}$ und Brüssel IIa (EuEheVO) $)^{85}$ genannten Verordnungen für Zivil- und Handelssachen sowie für Familiensachen. Die EuUnthVO, ${ }^{86}$ die EuErbVO ${ }^{87}$ sowie die geplante EhegüterrechtsVO ${ }^{88}$ vereinen Regeln zum IPR und zur internationalen Zuständigkeit, Anerkennung und Vollstreckung. Die Verordnungen über einen Europäischen Vollstreckungstitel ${ }^{89}$ und ein Europäisches Mahnverfahren ${ }^{90}$ versuchen, die grenzüberschreitende Forderungsbeitreibung noch weiter zu beschleunigen und zu vereinfachen. Darüber hinaus erstreckt sich die Gesetzgebungstätigkeit der EU bereits auf das Erkenntnisverfahren, siehe die VO über die Einführung eines europäischen Verfahrens für geringfügige Forderungen. ${ }^{91}$ Alle drei zuletzt genannten Verordnungen stellen Verfahren bereit, die alternativ zu denen der EuGVVO bzw. des mitgliedstaatlichen Rechts genutzt werden können. Schließlich spielen für die Praxis der grenzüberschreitenden Zivilprozesse die Europäische Zustellungsverordnung ${ }^{92}$ und die Europäische Beweisverordnung ${ }^{93}$ eine zentrale Rolle.

\section{Eine Europäische IPR-Kodifikation am Horizont?}

Nimmt man die existierenden und in Vorbereitung befindlichen Verordnungen zusammen und berücksichtigt weiter die de facto-Vereinheitlichung des internationalen Gesellschaftsrechts durch die Rechtsprechung des EuGH, ${ }^{94}$ so bleibt kaum ein Gebiet

84 Verordnung (EG) Nr. 44/2001 des Rates über die gerichtliche Zuständigkeit und die Anerkennung und Vollstreckung von Entscheidungen in Zivil- und Handelssachen, ABl. EG Nr. L 12 /1.

85 Verordnung (EG) Nr. 2201/2003 des Rates über die Zuständigkeit und die Anerkennung und Vollstreckung von Entscheidungen in Ehesachen und in Verfahren betreffend die elterliche Verantwortung v. 27.11.2007, ABl. EG Nr. L 338/1.

86 Fn. 83.

87 Fn. 81.

88 Fn. 82.

89 Verordnung (EG) Nr. 805/2004 des Europäischen Parlaments und des Rates zur Einführung eines europäischen Vollstreckungstitels für unbestrittene Forderungen, ABl.EG Nr. L 143/15.

90 Verordnung (EG) Nr. 1896/2006 des Europäischen Parlaments und des Rates zur Einführung eines Europäischen Mahnverfahrens, ABl.EG Nr. L 399/1.

91 Verordnung (EG) Nr. 861/2007 des Europäischen Parlaments und des Rates zur Einführung eines europäischen Verfahrens für geringfügige Forderungen, ABl.EG Nr. L 199/1.

92 Verordnung (EG) Nr. 1393/2007 des Europäischen Parlaments und des Rates über die Zustellung gerichtlicher und außergerichtlicher Schriftstücke in Zivil- und Handelssachen in den Mitgliedstaaten und zur Aufhebung der Verordnung (EG) Nr. 1348/2000, ABl. EU 2007 Nr. L 324/79.

93 Verordnung (EG) Nr. 1206/2001 des Rates über die Zusammenarbeit der Gerichte in den Mitgliedstaaten auf dem Gebiet der Beweisaufnahme in Zivil- und Handelssachen, ABl. EG 2001 Nr. L 174/1.

94 EuGH 27.9.1988, Rs. 81/87, The Queen/H.M. Treasury and Commissioners of Inland Revenue, ex parte Daily Mail and General Trust PLC, Slg. 1988, 5483; 9.3.1999, Rs. C-212/97, Centros Ltd./ Erhvervs- og Selskabsstyrelsen, Slg. 1999 I 1459; 5.11.2002, Rs. C-208/00, Überseering B.V./Nordic Construction Company Baumanagement GmbH (NCC), Slg. 2002 I 9919; 30.9.2003, Rs. C-167/01, Kamer van Koophandel en Fabrieken voor Amsterdam/Inspire Art Ltd., Slg. 2003 I 10155; 13.12.2005, Rs. C-411/03, SEVIC Systems Aktiengesellschaft/AG Neuwied, Slg. 2005 I 10805; 16.12.2008, Rs. C-210/06, Cartesio Oktató es Szolgáltató bt, Slg. 2009 I 09641; 12.7.2012, Rs. C-378/10, VALE, Slg. 2012 I 0000; vgl. zum Ganzen Ch. Teichmann, Gesellschaftsrecht im System der Europäischen Niederlassungsfreiheit, ZGR 2011, S. 639 ff. 
des internationalen Privatrechts von der Vergemeinschaftung ausgespart. Nur das Sachenrecht und Teile des Personen- und Familienrechts (Rechts- und Geschäftsfähigkeit, Eheschließung, allgemeine Ehewirkungen, Abstammung, Adoption, ElternKind-Verhältnis, Vormundschaft) sind bislang von der rechtssetzenden Tätigkeit der europäischen Institutionen ausgenommen, wobei aber einerseits zu beachten ist, dass diese Gebiete teilweise durch Haager Konventionen abgedeckt oder zumindest berührt werden (Adoptionsübereinkommen, ${ }^{95}$ Kinderschutzübereinkommen), ${ }^{96}$ teilweise der EuGH mittels eines aus der Unionsbürgerschaft abgeleiteten Anerkennungsprinzips eine faktische Vereinheitlichung herbeigeführt hat, so im Namensrecht. ${ }^{97}$

Damit liegt die Frage auf der Hand, ob die Zeit nicht reif ist, die einzelnen Verordnungen in einer einzigen europäischen IPR-Kodifikation zusammenzufassen, Unstimmigkeiten zwischen den Verordnungen zu bereinigen, letzte Lücken wie beispielsweise die Anknüpfung der Persönlichkeitsrechtsverletzungen zu füllen, und das Werk durch einen Allgemeinen Teil zu krönen. In der kollisionsrechtlichen Literatur wird die Frage mit tendenziell positiver Grundstimmung seit einiger Zeit diskutiert; ${ }^{98}$ ein erster Entwurf für einen Allgemeinen Teil aus der Feder von Paul Lagarde liegt bereits vor. ${ }^{99}$ Das Europäische Parlament, das schon 2010 Zustimmung zu solchen Plänen signalisiert hatte, ${ }^{100}$ hat kürzlich einen Auftrag für eine Machbarkeitsstudie an das Asser-Institut vergeben.

So sehr das Streben nach Zusammenführung, Beseitigung von Redundanzen, Systematisierung und Abrundung einleuchtet, ${ }^{101}$ bleibt die Realisierung doch mit erheblichen Schwierigkeiten behaftet, die aus dem unterschiedlichen räumlichen Geltungs-

95 Haager Übereinkommen über den Schutz von Kindern und die Zusammenarbeit auf dem Gebiet der internationalen Adoption, vom 29.5.1993, BGBl. 2001, II, S. 1035.

96 Haager Übereinkommen über die Zuständigkeit, das anzuwendende Recht, die Anerkennung, Vollstreckung und Zusammenarbeit auf dem Gebiet der elterlichen Verantwortung und der Maßnahmen zum Schutz von Kindern vom 19.10.1996, BGBl. 2009, II, S. 603.

97 EuGH 2.10.2003, Rs. C-148/02, Garcia Avello, Slg. 2003 I 11613; EuGH 14.10.2008, Rs. C-353/06, Grunkin Paul II, Slg. 2008 I 76.

98 M. Czepelak, Would We Like to Have a European Code of Private International Law?, ERPL 2010, S. 705 ff.; E. Jayme, Die Kodifikationsidee am Beispiel der Privatautonomie, in: B. Jud/ W. Rechberger/G. Reichelt (Hrsg.), Kollisionsrecht in der Europäischen Union, Jan Sramek Verlag, Wien 2008, S. 63 ff.; E.-M. Kieninger, Das Europäische IPR von der Kodifikation?, in: H. Kronke/K. Thorn (Hrsg.), FS von Hoffmann (Fn. 65), S. 184 ff.; K. Siehr, Die Kodifikation des Europäischen IPR Hindernisse, Aufgaben und Lösungen, in: B. Jud/ W. Rechberger/G. Reichelt (Hrsg.), aaO., S. 77 (93 ff.).

99 P. Lagarde, Embryon de Règlement portant Code Européen de Droit International Privé, RabelsZ 75 (2011), S. $673 \mathrm{ff}$.

100 Vgl. Entschließung vom 7. September zu der Umsetzung und Überprüfung der Verordnung (EG) Nr. 44/2001 des Rates über die gerichtliche Zuständigkeit und die Anerkennung und Vollstreckung von Entscheidungen in Zivil- und Handelssachen“ (2009/2140/INI), Ziffer 1: „... meint, dass letztlich eine umfassende Kodifizierung des internationalen Privatrechts angestrebt werden könnte“.

$101 \mathrm{Zu}$ den einzelnen Gründen, die für eine IPR-Kodifikation ins Feld geführt werden können, vgl. E.M. Kieninger, Das Europäische IPR von der Kodifikation?, in: H. Kronke/K. Thorn (Hrsg.), FS von Hoffmann (Fn. 65), S. $184 \mathrm{ff}$. 
bereich der bestehenden Verordnungen resultieren, die wiederum eine Folge der Besonderheiten des familienrechtlichen Gesetzgebungsverfahrens (Notwendigkeit der Einstimmigkeit im Rat) und der Sonderrolle Dänemarks, Großbritanniens und Irlands sind, und sich daher auch im Rahmen eines kodifikatorischen Ansatzes nicht ohne weiteres lösen lassen werden. ${ }^{102}$ Auch lässt sich mit Fug und Recht bezweifeln, ob sich gerade die beiden Mitgliedstaaten des Common Law für eine Kodifikationsidee werden begeistern lassen können. ${ }^{103}$

Die gegenwärtig vorstellbaren Alternativen sind einerseits eine Kernkodifikation für das Zivil- und Handelsrecht als „kleine Lösung“, bestehend aus einer Zusammenführung der Rom I- und Rom II- Verordnungen, eventuell ergänzt um die EuGVVO. In Bezug auf alle drei Texte haben das UK und Irland ihr opt-in erklärt; Dänemark ist an die EuGVVO durch Staatsvertrag gebunden; Übereinkommen zu den beiden Rom-Verordnungen werden nicht ausbleiben können, wenn sich Dänemark im internationalen Schuldrecht nicht völlig isolieren will. Mit der Zusammenführung von Rom I und Rom II könnte ein Einheitstext für das auf vertragliche und außervertragliche Schuldverhältnisse anwendbare Recht geschaffen werden, der Redundanzen vermeidet, etwa die Wiederholungen zum Renvoi, zum ordre public, zur Behandlung von Mehrrechtsstaaten und zur universellen Anwendbarkeit, und unnötige Abweichungen zwischen den beiden bestehenden Verordnungen, wie etwa in Bezug auf die Eindeutigkeit der Rechtswahl (vgl. Art. 3 Abs. 1 S. 1 Rom I-VO und Art. 14 Abs. 1 S. 2 Rom II-VO) oder die Berücksichtigung von Eingriffsnormen (Art. 9 Rom I-VO und Art. 16 Rom II-VO) bereinigt. Die Hinzufügung der EuGVVO würde dem Trend der neueren Verordnungen bzw. Verordnungsvorschläge zum Unterhaltsrecht, zum Erbrecht und zum Ehegüterrecht entsprechen, die jeweils für ihr Sachgebiet das anwendbare Recht gemeinsam mit der Gerichtszuständigkeit und der Anerkennung und Vollstreckung regeln. Diese Einteilung nach Sachgebieten hat den praktischen Vorteil, dass der mit einem Erb- oder Ehegüterrechtstreit befasste Praktiker nur einen einzigen Text zu Rate ziehen muss, um anwendbares Recht, internationale Zuständigkeit und die Chancen einer Anerkennung und Vollstreckung in anderen Mitgliedstaaten beurteilen zu können. Freilich reicht die EuGVVO sachlich weit über das Kernzivilrecht der Schuldverhältnisse hinaus und erfasst insbesondere im Katalog der ausschließlichen Zuständigkeiten (vgl. Art. 22 EuGVVO) auch Gesellschafts- und Sachenrecht, also Materien, die in den Rom I und II-VO'en nicht berührt sind. Die Zusammenführung könnte für diese Materien wieder zu einer praktischen Erschwerung des Auffindens der richtigen Rechtsquelle führen.

102 Ausführlich R. Wagner, in: S. Leible/H. Unberath (Hrsg.), Brauchen wir eine Rom 0-Verordnung?, in Vorbereitung.

103 Vgl. P. North, Problems of Codification in a Common Law System, RabelsZ 46 (1982), S. 490 (500 ff.). 
Eine zweite Alternative wäre die Zusammenführung aller Verordnungen zum IPR und IZVR, auch derjenigen zum Familien- und Erbrecht, aber unter Aussparung der spezielleren zivilprozessrechtlichen Verordnungen (EuVTVO, EuMahnVO, EuGFVO), um das Unternehmen nicht zu überfrachten. Eine solche „große Lösung“ wäre aber wohl nur um den Preis zu erzielen, dass neben Dänemark auch das Vereinigte Königreich und Irland, möglicherweise auch noch weitere Staaten wie etwa Schweden, die der Rom III-Verordnung ferngeblieben sind, außen vor blieben. Manche Verordnungen, wie Rom I und II, würden dann getrennte Wege gehen: In einigen Mitgliedstaaten würden sie in der bisherigen Einzelfassung weiter in Kraft bleiben, in anderen würde eine integrierte und damit auch reformierte Fassung an deren Stelle treten, mit allen Schwierigkeiten, die das für die praktische Rechtsanwendung mit sich bringt. Allenfalls die Hoffnung auf eine Vereinheitlichungs- und Reformdynamik, der sich die zunächst fernbleibenden Mitgliedstaaten nicht auf Dauer entziehen könnten, vermag eine solche neue Rechtszersplitterung als verantwortbar erscheinen lassen.

Eine dritte Alternative wäre die Ausgliederung der „allgemeinen“ Vorschriften aus den bestehenden Verordnungen und ihre Integration in einen Allgemeinen Teil, eine sogenannte „Rom 0-VO“. ${ }^{104}$ Allerdings hat das im Juli 2012 unter der Leitung von Stefan Leible und Hannes Unberath veranstaltete Bayreuther Symposium mit dem Titel „Brauchen wir eine Rom 0-Verordnung?“ eher ernüchternde Ergebnisse gezeitigt: Abgesehen von der unproblematischen Übernahme der schon bisher gleichlautenden Vorschriften zur universellen Anwendbarkeit, zur Behandlung von Mehrrechtsstaaten oder zum ordre-public-Vorbehalt konnten als geeignete Kandidaten für eine Auslagerung in eine Verordnung zum Allgemeinen Teil nur die Behandlung der Eingriffsnormen und die bisher in den einschlägigen Verordnungen noch gar nicht behandelte Frage nach der Rolle der Parteiautonomie bei der Ermittlung und Anwendung des fremden Rechts identifiziert werden. Der letzte Punkt wird freilich allgemein als entscheidend für die Verwirklichung des Ziels der Europäischen Kollisionsrechtsvereinheitlichung betrachtet, ${ }^{105}$ denn wenn es in manchen Mitgliedstaaten den Parteien überlassen bleibt, die Anwendung des Kollisionsrechts durch das Gericht zu verlangen und Beweis über den Inhalt das anzuwendenden ausländischen Rechts zu erbringen, in anderen dagegen beides ex officio geschieht, so ist eine einheitliche Anwendung der europaweit einheitlichen IPR-Regeln Utopie - die Vereinheitlichung findet nur auf dem Papier statt. So dringlich daher gerade hier eine ein-

104 Dazu S. Leible/H. Unberath (Hrsg.) (Fn. 102).

105 Vgl. C. Trautmann, Europäisches Kollisionsrecht und ausländisches Recht im nationalen Zivilverfahren, Tübingen 2011; C. Esplugues, General Report on the Application of Foreign Law by Judicial and Non-Judicial Authorities in Europe, in: ders./J. Iglesias/G. Palao (eds.), Application of Foreign Law, München 2011, S. 3 ff.; A. Flessner, Das ausländische Recht im Zivilprozess - die europäischen Anforderungen, in: G. Reichelt (Hrsg.), 30 Jahre österreichisches IPR-Gesetz - Europäische Perspektiven, Manz'sche Verlags- und Universitätsbuchhandlung Wien, 2009, S. 35 (42). 
heitliche Lösung wäre, ${ }^{106}$ so schwierig gestaltet sie sich aber auch, weil dabei grundsätzliche Vorstellungen von der Rollenverteilung zwischen Parteien und Gericht angesprochen sind, die man schon beinahe zum rechtskulturellen Erbe zählen kann. Trevor Hartley schließt seine rechtsvergleichende Übersicht zu „Pleading and Proof of Foreign Law" 107 mit der suggestiven Frage: ${ }^{108}$

"Is the function of a judge to ascertain "the truth" and to "do justice" in the abstract, or is it to decide the case according to the pleadings and evidence? [...] The heavy hand of history, therefore, is likely to ensure that the legal systems of England and the Continent retain their distinctive features for many years to come."

Auch bei dieser Alternative ist mithin fast zwingend davon auszugehen, dass das Unternehmen ohne Großbritannien und Irland stattfinden würde.

\section{Vergleich}

Auch wenn eine IPR-Kodifikation damit gewiss noch nicht unmittelbar vor der Tür steht, so schlägt ihr doch eine wesentlich größere Sympathie entgegen als der Idee eines EuZGB. Weiterhin lässt sich feststellen, dass sich die europäische Vereinheitlichung des Kollisionsrechts mit einer wesentlich größeren Stringenz und Kontinuität entwickelt hat als die Angleichung des materiellen Zivilrechts. Selbst ohne eine veritable Kodifikation sind faktisch weite Teile des Kollisionsrechts und des Internationalen Zivilverfahrensrechts vereinheitlicht, während sich die materielle Zivilrechtsangleichung noch immer im Wesentlichen auf das Verbrauchervertragsrecht beschränkt. ${ }^{109}$ Verschiedene Ursachen kommen als Erklärung in Betracht:

\section{Kontinuität}

Zum einen spielt sicher die bereits erwähnte Kontinuität eine Rolle. Das einheitliche IPR und IZVR stand zunächst ganz in der bewährten Tradition der kollisionsrechtsvereinheitlichenden Staatsverträge, wie sie seit Jahrzehnten unter dem Dach der Haager Konferenz für Internationales Privatrecht ausgearbeitet worden sind und noch werden. Auch nach der Übertragung der Kompetenz auf die EU änderte sich das nicht. Die Rom I-VO nahm nur behutsame Korrekturen vor - „Evolution statt

106 Zu den verschiedenen Lösungsmöglichkeiten E.-M. Kieninger, Ermittlung und Anwendung ausländischen Rechts, in: S. Leible/H. Unberath (Hrsg.) (Fn. 102).

107 T. Hartley, Pleading and Proof of Foreign Law: The Major European Systems Compared, International and Comparative Law Quarterly 45 (1996), S. 271 (292).

108 T. Hartley, Pleading (Fn. 107), S. 271 (292).

109 Sofern man sich auf das Zivilrecht im Sinne der fünf Bücher des BGB beschränkt und vom Gesellschafts-, Arbeits-, Immaterialgüter- und Wettbewerbsrecht absieht. 
Revolution“, so wurde das neue Instrument allgemein charakterisiert. ${ }^{110}$ Eine „Conflicts Revolution“, wie sie in den USA in den 1960er und 70er Jahren stattgefunden hatte, gab und gibt es mit der Europäisierung des IPR nicht. ${ }^{111}$ Die Rom III-VO und die EuErbVO brachten zwar mit der Zurückdrängung der in Deutschland und anderen kontinentaleuropäischen Ländern bis dahin noch vorherrschenden Anknüpfung an die Staatsangehörigkeit sowie einer Stärkung der Privatautonomie gewichtigere Änderungen mit sich, nahmen aber letztlich nur Strömungen auf, die in der Haager Konferenz das internationale Familienrecht schon länger dominierten.

\section{Rechtsprechung des EuGH}

Der EuGH als neue Instanz zur Wahrung einer einheitlichen Auslegung kam zunächst nur in Bezug auf das Brüsseler Übereinkommen zum Einsatz, denn das Protokoll zum Römer Übereinkommen von 1980, das ihm die Auslegungskompetenz hätte übertragen sollen, trat erst am 1.8.2004 in Kraft. Sorgen, der EuGH könne sich zur kollisionsrechtlichen Superrevisionsinstanz aufschwingen, existierten zu keiner Zeit. Die Bedenken gingen eher in die andere Richtung, als mit dem Amsterdamer Vertrag die Vorlagemöglichkeit für die unter Titel IV verabschiedeten Maßnahmen auf die letztinstanzlichen Gerichte beschränkt wurde. ${ }^{112}$ Der Lissabonner Vertrag hat diesen allgemein als Fehlentwicklung betrachteten Schritt wieder zurückgenommen. Die Rechtsprechung des Gerichtshofs zum EuGVÜ und zur EuGVVO (Rechtsprechung zu den Rom-Verordnungen gibt es bislang kaum) orientiert sich im Großen und Ganzen an den Fixsternen Rechtssicherheit, Vorhersehbarkeit und engster Verbindung ${ }^{113}$ und bewegt sich damit ebenfalls in bewährten Bahnen. Spektakulärer und umstrittener waren die Entscheidungen zum internationalen Gesellschaftsrecht, ${ }^{114}$

110 S. Leible/M. Lehmann, Die Verordnung über das auf vertragliche Schuldverhältnisse anzuwendende Recht (Rom I), RIW 2008, S. 528, 529; ähnlich auch P. Mankowski, Der Vorschlag für eine Rom IVerordnung, IPRax 2006, S. 101: „,evolutionärer Ansatz; behutsame Entwicklung“.

111 W.-H. Roth, Europäische Kollisionsrechtsvereinheitlichung - Überblick - Kompetenzen - Grundfragen, in: E.-M. Kieninger/O. Remien (Hrsg.) (Fn. 69), S. 11 (27 f.). A.A. R. Michaels, Die europäische IPR-Revolution, in: D. Baetge/J. von Hein/M. von Hinden, FS Kropholler (Fn. 65), S. 151 ff., der allerdings weniger auf die Vereinheitlichung durch Verordnungen als die Rechtsprechung des EuGH zum Namensrecht und die dadurch ausgelöste Diskussion um ein neues „Anerkennungsprinzip“ zielt, das die klassische IPR-Methode verdrängen könnte; vgl. dazu D. Henrich, Anerkennung statt IPR: Eine Grundsatzfrage, IPRax 2005, S. 422 ff.; P. Lagarde, Développements futurs du droit international privé dans une Europe en voie d'unification: quelques conjectures, RabelsZ 68 (2004), S. 225 ff.; H. P. Mansel, Anerkennung als Grundprinzip des Europäischen Rechtsraums, Zur Herausbildung eines Europäischen Anerkennungs-Kollisionsrechts: Anerkennung statt Verweisung als neues Strukturprinzip des Europäischen internationalen Privatrechts?, RabelsZ 70 (2006), S. 651 ff.; H. J. Sonnenberger, Anerkennung statt Verweisung? Eine neue internationalprivatrechtliche Methode?, in: J. Bernreuther/R. Freitag/S. Leible et al. (Hrsg.), FS Spellenberg (Fn. 78), S. 371 ff.

112 Vgl. J. Kropholler, Zivilprozessrecht (Fn. 73), Einl Rdn. 34 m.w.N.

113 Näher E.-M. Kieninger, Die Rolle des EuGH nach Inkrafttreten der „Rom-Verordnungen“, in: P.Chr. Müller-Graff/S. Schmahl/V. Skouris (Hrsg.), Europäisches Recht zwischen Bewährung und Wandel, Festschrift für Dieter Scheuing, Baden-Baden 2011, S. 110 (116 ff.).

114 Oben Fn. 94. 
zum Namensrecht ${ }^{115}$ und zur Qualifikation des Entschädigungsanspruchs nach der Handelsvertreterrichtlinie als international zwingend. ${ }^{116}$ Diese Entscheidungen ergingen aber sämtlich nicht auf der Grundlage kollisionsrechtsvereinheitlichender Verordnungen, sondern unter Zugrundelegung des Primärrechts bzw. von sachrechtsangleichenden Richtlinien und waren damit binnenmarktfinal ausgerichtet. Diese einseitige Ausrichtung hat der EU-Gesetzgeber in den Sekundärrechtsakten zum Kollisionsrecht, die ja alle universell anwendbar sind, also auch dann, wenn der grenzüberschreitende Bezug nur zu Drittstaaten besteht, nicht weiter verfolgt. Kritik, wie sie etwa an der Putz und Weber Entscheidung des EuGH geübt worden ist, ${ }^{117}$ blieb dem EuGH in Bezug auf die Auslegung des vereinheitlichten IPR und IZVR bisher erspart.

\section{Immanente Beschränkung auf grenzüberschreitenden Sachverhalte}

Ein weiterer Vorzug der Koordinierung des Zivilrechts durch vereinheitlichtes IPR ist die immanente Beschränkung dieser Maßnahmen auf grenzüberschreitende Sachverhalte. Hier leuchtet die Notwendigkeit einer Vereinheitlichung unmittelbar ein, während sie für gesetzgeberische Akte, die auch rein nationale Vorgänge erfassen sollen, wie insbesondere die Vertragsrechtsharmonisierungsrichtlinien viel schwerer zu begründen ist. Die Frage, ob ein einheitliches europäisches Vertragsrecht nur für grenzüberschreitende Verträge wählbar bzw. bei einem opt-out Modell von vorneherein auf solche Verträge beschränkt bleiben sollte, spaltet bis heute die Gemüter; ${ }^{118}$ die Kommission hat sich im Wege des Kompromisses in ihrem CESL-Vorschlag für ein Mitgliedstaatenwahlrecht entschieden.

\section{IPR als politikfernes Spezialistenfach}

Ein weiterer, möglicherweise entscheidender Grund für die weitgehend kontinuierliche und ungestörte Entfaltung des europäischen IPR und IZVR ist seine Komplexität und daraus folgende Politikferne. Natürlich gilt das nicht im selben Maß für alle Fragen. So ist etwa im internationalen Gesellschaftsrecht durchaus auch im politischen Raum diskutiert worden, ob der pauschale Übergang zur Anknüpfung an den Satzungssitz bzw. Registrierungsort nicht zu einer Aushebelung der deutschen Vorschriften über die unternehmerische Mitbestimmung führen könnte. Der Refe-

115 Oben Fn. 97.

116 EuGH 9. 11. 2000, Rs. C-381/98, Ingmar GB Ltd./Eaton Leonard Technologies Inc., Slg. 2000 I, 9305; dazu R. Freitag/S. Leible, Der internationale Anwendungsbereich der Handelsvertreterrichtlinie - Europäisches Handelsvertreterrecht weltweit?, RIW 2001, S. $287 \mathrm{ff}$.

117 Oben Fn. 17 sowie D. Kaiser, EuGH zum Austausch mangelhafter eingebauter Verbrauchsgüter, JZ 2011, S. $978 \mathrm{ff}$.

118 Vgl. dazu bereits U. Drobnig, Ein Vertragsrecht für Europa, in: F. Baur/K. J. Hopt/ K. P. Mailänder (Hrsg.), Festschrift für Ernst Steindorff zum 70. Geburtstag, Berlin 1990, S. 1141 ff.; für einen weiten Anwendungsbereich des CESL H. Eidenmüller/N. Jansen/E.-M. Kieninger/G. Wagner/R. Zimmermann, Vorschlag (Fn. 55), JZ 2012, S. 269 (275). 
rentenentwurf, ${ }^{119}$ der ganz wesentlich auf Vorarbeiten des Deutschen Rates für Internationales Privatrecht ${ }^{120}$ basierte und wie diese auch eine Blaupause für eine Regelung auf europäischer Ebene hätte sein können, wurde aus den genannten Gründen bislang politisch nicht weiter verfolgt, obwohl er über die Mitbestimmung kein Wort verliert. Im Allgemeinen aber lässt sich mit Fragen wie der, ob auch ausländischen Eingriffsnormen im Forumstaat Wirkung verliehen werden sollte, oder ob in Verbraucherverträgen eine Rechtswahl strikt ausgeschlossen oder einem Günstigkeitsvergleich mit dem objektiven Vertragsstatut unterworfen werden sollte, oder ob im internationalen Erbrecht der Renvoi anders als im Schuldrecht beschränkt zugelassen werden sollte, kein Staat machen. Selbst das überwältigende Votum des 68. Deutschen Juristentages gegen eine Abkehr von der Staatsangehörigkeitsanknüpfung im internationalen Erbrecht ${ }^{121}$ hat keinerlei Spuren in der europäischen Gesetzgebungstätigkeit hinterlassen. Ein Aufschrei unter dem Motto „Rettet das EGBGB vor Brüssel“122 ist noch in keiner Tageszeitung veröffentlicht worden.

\section{Koordinierung des Privatrechts durch vereinheitlichtes IPR und IZVR als Ausdruck einer noch unvollkommenen Europäischen Union}

Möglicherweise entspricht die Koordinierung der grenzüberschreitenden privatrechtlichen Beziehungen in der EU, seien sie vermögens-, familien- oder erbrechtlicher Natur, durch vereinheitlichtes IPR und IZVR und nicht durch einheitliches materielles Recht dem gegenwärtigen Stand der Integration auch im übrigen politischen Raum. ${ }^{123}$ Die EU ist kein Bundesstaat mit zentraler Regierung; die demokratische Legitimation ihrer Rechtsakte ist trotz der Erweiterung der Mitentscheidungsrechte des Europäischen Parlaments noch immer deutlich schwächer als die der mitgliedstaatlichen Gesetzgebung. Die Kompetenz zu einer flächendeckenden Vereinheitlichung des materiellen Zivilrechts unter Verdrängung der nationalen Kodifikationen

119 Referentenentwurf für ein Gesetz zum Internationalen Privatrecht der Gesellschaften, Vereine und juristischen Personen, siehe dazu R. Wagner/B. Timm, Der Referentenentwurf eines Gesetzes zum Internationalen Privatrecht der Gesellschaften, Vereine und juristischen Personen, IPRax 2008, S. 81 ff.; G. Roth, Eine europäische Initiative zur Kodifizierung der Gründungstheorie, RdW 2007, S. 206 ff.; J. Clausnitzer, Die Novelle des Internationalen Gesellschaftsrechts, NZG 2008, S. 321 ff.; P. Bollacher, Referentenentwurf zur Regelung des Internationalen Gesellschaftsrechts, RIW 2008, S. 200 ff.; M. Rotheimer, Referentenentwurf zum Internationalen Gesellschaftsrecht, NZG 2008, S. $181 \mathrm{f}$.

120 H. J. Sonnenberger (Hrsg.), Vorschläge und Berichte zur Reform des europäischen und deutschen internationalen Gesellschaftsrechts, Tübingen 2007.

121 Verhandlungen des 68. Deutschen Juristentages, Bd. II/1, Zivilrechtliche Abteilung, L 154.

122 Vgl. aber Fn. 39.

123 Vgl. dazu bereits J. Taupitz, Privatrechts- oder Kollisionsrechtsvereinheitlichung in Europa?, 1994, Zentrum für Europäisches Wirtschaftsrecht, Vorträge und Berichte Nr. 44. 
ist nach dem gegenwärtigen Stand des Primärrechts nicht gegeben; ${ }^{124}$ der politische Wille hierfür ohnehin nicht sichtbar. Sollte die gegenwärtige Wirtschafts- und Finanzkrise hingegen zu einer Vertiefung der Integration, einer Ausweitung der Kompetenzen der Union und - damit einhergehend - zu einer noch stärkeren Demokratisierung ihrer Institutionen führen, ${ }^{125}$ so ist möglicherweise auch die Zeit reif für eine zivilrechtliche Kodifikation als sichtbarem Ausdruck der europäischen Staatlichkeit, ähnlich den großen nationalen Kodifikationen. ${ }^{126}$ Bis dahin bleibt reichlich Zeit, einen solchen großen Wurf wissenschaftlich vorzubereiten.

124 Vgl. statt aller M. Schmidt-Kessel, Europäisches Zivilgesetzbuch, in: J. Basedow et al. (Hrsg.), Handwörterbuch (Fn. 2), S. 551 (554) unter 5.; eine Kompetenz aus Art. 114 oder 352 AEUV für spezifischer zugeschnittene Projekte dürfte dagegen bestehen, etwa für einen auf das (allgemeine) Vertragsrecht, die Einführung einer Eurohypothek oder eines Europäischen Kreditsicherungsrechts begrenzten Gesetzgebungsakt, vgl. J. Basedow, Über Privatrechtsvereinheitlichung und Marktintegration, in: U. Immenga/W. Möschel/D. Reuter (Hrsg.), Festschrift für Ernst-Joachim Mestmäcker, Baden-Baden 1996, S. 347 (358 ff.); W.-H. Roth, Secured Credit and the Internal Market: The Fundamental Freedoms and the EU's Mandate for Legislation, in: H. Eidenmüller/E.-M. Kieninger (eds.), The Future of Secured Credit in Europe, Berlin 2008, S. 36 (61 ff.).

125 Vgl. J. Habermas, Die Krise der Europäischen Union im Lichte einer Konstitutionalisierung des Völkerrechts - Ein Essay zur Verfassung Europas, in: ders., Zur Verfassung Europas, Berlin 2011, S. 39 (48 ff.).

126 O. Lando, Comments and Questions Relating to the European Commission's Proposal for a Regulation on a Common European Sales Law, ERPL 2011, S. 717 (728). 